\title{
An Extension of the Fuzzy Possibilistic Clustering Algorithm Using Type-2 Fuzzy Logic Techniques
}

\author{
Elid Rubio, Oscar Castillo, Fevrier Valdez, Patricia Melin, \\ Claudia I. Gonzalez, and Gabriela Martinez
}

Tijuana Institute of Technology, Tijuana, BC, Mexico

Correspondence should be addressed to Oscar Castillo; ocastillo@tectijuana.mx

Received 20 July 2016; Accepted 9 January 2017; Published 31 January 2017

Academic Editor: Ning Xiong

Copyright (c) 2017 Elid Rubio et al. This is an open access article distributed under the Creative Commons Attribution License, which permits unrestricted use, distribution, and reproduction in any medium, provided the original work is properly cited.

\begin{abstract}
In this work an extension of the Fuzzy Possibilistic C-Means (FPCM) algorithm using Type-2 Fuzzy Logic Techniques is presented, and this is done in order to improve the efficiency of FPCM algorithm. With the purpose of observing the performance of the proposal against the Interval Type-2 Fuzzy C-Means algorithm, several experiments were made using both algorithms with well-known datasets, such as Wine, WDBC, Iris Flower, Ionosphere, Abalone, and Cover type. In addition some experiments were performed using another set of test images to observe the behavior of both of the above-mentioned algorithms in image preprocessing. Some comparisons are performed between the proposed algorithm and the Interval Type-2 Fuzzy C-Means (IT2FCM) algorithm to observe if the proposed approach has better performance than this algorithm.
\end{abstract}

\section{Introduction}

Different areas of research have widely used clustering algorithms for different purposes, such as image segmentation $[1,2]$, data mining [3], pattern recognition [4], classification [5], and modeling [6]. Clustering algorithms arise due to need to find data groups that share similar features in a given dataset; at this time there are several fuzzy clustering algorithms, such as FCM [4], PCM [7], FPCM [8], and PFCM [8]. The acceptance of these algorithms is due to the fact that they permit a datum to belong to different data clusters into a given dataset.

However, the algorithms mentioned above do not have the capability to handle the uncertainty that lies within a dataset during the clustering process; because of this, some of these algorithms (FCM and PCM) have been improved using Type-2 Fuzzy Logic Techniques $[9,10]$, and the improvement of these algorithms has been called Interval Type-2 Fuzzy CMeans (IT2FCM) [11, 12] and Interval Type-2 Possibilistic CMeans (IT2PCM) $[12,13]$, respectively. These algorithms have been used for different purposes, such as modeling [14-17], creation of membership functions $[18,19]$, image processing $[20,21]$, and classification [22]. In recent years research has also been performed in the extension of other clustering algorithms using Type-2 Fuzzy Logic Techniques, such as the ones proposed in [13, 23-27].

In this work we are presenting the extension of the FPCM using Type-2 Fuzzy Logic Techniques to provide this method with the capability of handling a higher degree of uncertainty in a dataset to solve real world problems where data clustering is involved. Other clustering algorithms have been extended using Type-2 Fuzzy Logic Techniques, but the FPCM algorithm has not been previously extended using these techniques.

This paper is organized as follows. Section 2 describes the extension of the FPCM algorithm presented in this paper, Section 3 shows the concept of cluster validation index to measure the performance of the clustering algorithm, Section 4 shows the results obtained by the IT2FPCM algorithm and its comparison with the IT2FCM algorithm, and Section 5 contains the conclusions and future work.

\section{Interval Type-2 Fuzzy Possibilistic C-Means Algorithm}

This is an extension of the FPCM algorithm proposed by N. R. Pal et al. in 1997, using Type-2 Fuzzy Logic Techniques, and 
in the same way that FPCM algorithm produces membership and possibilities using the weight exponents $m$ and $\eta$ for the fuzziness and possibility, respectively, this may now be represented by a range rather than a precise value; that is, $m=\left[m_{1}, m_{2}\right]$, where $m_{1}$ and $m_{2}$ represent the lower and upper limit of weighting exponent for fuzziness and $\eta=\left[\eta_{1}\right.$, $\eta_{2}$ ], where $\eta_{1}$ and $\eta_{2}$ represent the lower and upper limit of weighting exponent for possibility.

Because the $m$ value is represented by an interval, the fuzzy partition matrix $\mu_{i}\left(x_{k}\right)$ must be calculated for the interval $\left[m_{1}, m_{2}\right]$; for this reason $\mu_{i}\left(x_{k}\right)$ would be given by the belonging interval $\left[\underline{\mu}_{i}\left(x_{k}\right), \bar{\mu}_{i}\left(x_{k}\right)\right]$, where $\underline{\mu}_{i}\left(x_{k}\right)$ and $\bar{\mu}_{i}\left(x_{k}\right)$ represent the lower and upper limit of the belonging interval of datum $x_{j}$ to a clustering $v_{i}$, and updating the lower and upper limits of the range of the fuzzy membership matrix can be expressed as

$$
\begin{gathered}
\underline{\mu}_{i}\left(x_{k}\right)=\min \left\{\left(\sum_{j=1}^{c}\left(\frac{d_{i k}}{d_{j k}}\right)^{2 /\left(m_{1}-1\right)}\right)^{-1},\right. \\
\left.\left(\sum_{j=1}^{c}\left(\frac{d_{i k}}{d_{j k}}\right)^{2 /\left(m_{2}-1\right)}\right)^{-1}\right\}, \\
\bar{\mu}_{i}\left(x_{k}\right)=\max \left\{\left(\sum_{j=1}^{c}\left(\frac{d_{i k}}{d_{j k}}\right)^{2 /\left(m_{1}-1\right)}\right)^{-1},\right. \\
\left.\left(\sum_{j=1}^{c}\left(\frac{d_{i k}}{d_{j k}}\right)^{2 /\left(m_{2}-1\right)}\right)^{-1}\right\} .
\end{gathered}
$$

Because the $\eta$ value is represented by an interval, the possibilistic partition matrix $\tau_{i}\left(x_{k}\right)$ must be calculated for the interval $\left[\eta_{1}, \eta_{2}\right]$, and for this reason $\tau_{i}\left(x_{k}\right)$ would be given by the belonging interval $\left[\underline{\tau}_{i}\left(x_{k}\right), \bar{\tau}_{i}\left(x_{k}\right)\right]$, where $\underline{\tau}_{i}\left(x_{k}\right)$ and $\bar{\tau}_{i}\left(x_{k}\right)$ represent the lower and upper limit of the belonging interval of datum $x_{j}$ to a clustering $v_{i}$, and the update of the lower and upper limits of the range of the fuzzy membership matrix can be expressed as

$$
\begin{gathered}
\underline{\tau}_{i}\left(x_{k}\right)=\min \left\{\left(\sum_{j=1}^{c}\left(\frac{d_{i k}}{d_{j k}}\right)^{2 /\left(\eta_{1}-1\right)}\right)^{-1},\right. \\
\left.\left(\sum_{j=1}^{c}\left(\frac{d_{i k}}{d_{j k}}\right)^{2 /\left(\eta_{2}-1\right)}\right)^{-1}\right\}, \\
\bar{\tau}_{i}\left(x_{k}\right)=\max \left\{\left(\sum_{j=1}^{c}\left(\frac{d_{i k}}{d_{j k}}\right)^{2 /\left(\eta_{1}-1\right)}\right)^{-1},\right. \\
\left.\left(\sum_{j=1}^{c}\left(\frac{d_{i k}}{d_{j k}}\right)^{2 /\left(\eta_{2}-1\right)}\right)^{-1}\right\} .
\end{gathered}
$$

Updating the positions of the centroids of clusters should take into account the degree of belonging interval of the fuzzy and possibilistic matrices, resulting in a range of coordinates of the positions of the centroids of the clusters. The procedure for updating cluster prototypes in IT2FPCM requires calculating the centroids for the lower and upper of the limit of the interval using the fuzzy and possibilistic membership matrices, and these centroids are given by the following equations:

$$
\begin{aligned}
& \underline{v}_{i}=\frac{\sum_{j=1}^{n}\left(\underline{\mu}_{i}\left(x_{j}\right)+\underline{\tau}_{i}\left(x_{j}\right)\right)^{m_{1}} x_{j}}{\sum_{j=1}^{n}\left(\underline{\mu}_{i}\left(x_{j}\right)+\underline{\tau}_{i}\left(x_{j}\right)\right)^{m_{1}}}, \\
& \bar{v}_{i}=\frac{\sum_{j=1}^{n}\left(\bar{\mu}_{i}\left(x_{j}\right)+\bar{\tau}_{i}\left(x_{k}\right)\right)^{m_{1}} x_{j}}{\sum_{j=1}^{n}\left(\bar{\mu}_{i}\left(x_{j}\right)+\bar{\tau}_{i}\left(x_{k}\right)\right)^{m_{1}}} .
\end{aligned}
$$

The centroid calculation for the lower and upper limits of the interval results in an interval of coordinates of positions of the clusters centroids. Type-reduction and defuzzification use the type-2 fuzzy operations. The centroids matrix and the fuzzy partition matrix are obtained by the type-reduction operation as shown in the following equations:

$$
\begin{gathered}
v_{j}=\frac{\underline{v}_{j}+\bar{v}_{j}}{2}, \\
\mu_{i}\left(x_{j}\right)=\frac{\underline{\mu}_{i}\left(x_{j}\right)+\bar{\mu}_{i}\left(x_{j}\right)}{2} .
\end{gathered}
$$

This extension on the FPCM algorithm is intended to show that this algorithm is capable of handling uncertainty and is less susceptible to noise. Figure 3 shows the graphical representation of the steps FPCM algorithm in a block diagram where we can appreciate the operation of the Fuzzy Possibilistic C-Means algorithm step by step.

\section{Cluster Validation}

Cluster validation is one of the main topics in data clustering; this problem consists in finding and objective criterion to determine how good a partition generated by the clustering algorithm is. Nowadays there exist several index validation methods mentioned in [28-32], but these indices are proposed for validation of clusters found by Type-1 Fuzzy clustering algorithms. In order to evaluate the lower and upper bound of the interval of clusters found by the IT2FPCM and IT2FCM algorithms with some of the these indices of validation, we need to modify the following indices of validation to evaluate the partitions found by the Interval Type-2 Fuzzy clustering proposed in this work:

(i) Partition entropy index,

(ii) Xie-Beni Index,

(iii) MPE-DMFP index.

The partition entropy was proposed by Bezdek $[2,5,6]$ as a validation index for the Fuzzy C-Means algorithm and was defined by the following equation:

$$
\mathrm{PE}=-\frac{1}{n} \sum_{i=1}^{c} \sum_{j=1}^{n} u_{i j} \log _{2} u_{i j} .
$$


In a general we can define an optimal number of clusters $c^{*}$ with the solution $\min 2 \leq c \leq n-1$ for $\mathrm{PE}$ to produce a better performance by grouping the dataset $X$. To make this index able to evaluate the lower and upper bounds we need to compute the following equations to the upper and lower bounds, respectively:

$$
\begin{aligned}
& \mathrm{PE}^{\text {lower }}=-\frac{1}{n} \sum_{i=1}^{c} \sum_{j=1}^{n} \underline{u}_{i j} \log _{2} \underline{u}_{i j}, \\
& \mathrm{PE}^{\text {upper }}=-\frac{1}{n} \sum_{i=1}^{c} \sum_{j=1}^{n} \bar{u}_{i j} \log _{2} \bar{u}_{i j} .
\end{aligned}
$$

Xie and Beni in 1991 proposed a validation index based on compactness and separation $[2,5,6]$, which is defined by the following equation:

$$
\mathrm{XB}=\frac{\sum_{i=1}^{c} \sum_{j=1}^{n} u_{i j}^{m}\left\|x_{j}-v_{i}\right\|^{2}}{n \cdot \min _{\substack{i k \\ i \neq k}}\left\|v_{i}-v_{k}\right\|} .
$$

In general, an optimal number of clusters $c^{*}$ is found by solving min $2 \leq c \leq n-1$ for $\mathrm{XB}$ to produce a better clustering performance for the dataset $X$. To make this index able to evaluate the lower and upper bounds we compute the following equations to the upper and lower bounds, respectively:

$$
\begin{gathered}
\mathrm{XB}^{\text {lower }}=\frac{\sum_{i=1}^{c} \sum_{j=1}^{n} \underline{u}_{i j}^{m}\left\|x_{j}-\underline{v}_{i}\right\|^{2}}{n \cdot \min _{\substack{i k \\
i \neq k}}\left\|\underline{v}_{i}-\underline{v}_{k}\right\|}, \\
\mathrm{XB}^{\text {upper }}=\frac{\sum_{i=1}^{c} \sum_{j=1}^{n} \bar{u}_{i j}^{m}\left\|x_{j}-\bar{v}_{i}\right\|^{2}}{n \cdot \min _{\substack{i k \\
i \neq k}}\left\|\bar{v}_{i}-\bar{v}_{k}\right\|} .
\end{gathered}
$$

Elid Rubio et al. proposed the MPD-DFP index, which is composed of two metrics, the modified partition entropy index and the sum of the distances between the means of the fuzzy partitions. This validation index is represented by the following equation:

$$
\mathrm{MPE}-\mathrm{DMPF}=I_{\mathrm{MPE}}+D_{M},
$$

where the modified partition entropy $I_{\mathrm{MPE}}$ that represents the variation of the data in clusters of the dataset is represented by the following equations:

$$
I_{\mathrm{MPE}}=-\frac{1}{n} \sum_{i=1}^{c} \sum_{k=1}^{n} u_{i j}^{2} \log _{2} u_{i j}
$$

And the sum of the distances between the means of the fuzzy partition $D_{M_{k}}$ that represents the separation between clusters in the dataset

$$
D_{M_{k}}=\sum_{\substack{i, j=1 \\ i \neq j}}^{k}\left\|M_{i}-M_{j}\right\|^{2}, \quad k=1, \ldots, c,
$$

where $M_{k}$ is the mean of the fuzzy partitions generated by the Fuzzy C-Means algorithm. In general, we can define an optimal number of clusters $c^{*}$ for the solution $\min 2 \leq c \leq n-$ $1 I_{\mathrm{MPE}-\mathrm{DMFP}}$ to produce a better performance by grouping the dataset $X$. To make this index able to evaluate the lower and upper bounds of the interval cluster we compute the following equations to the upper and lower bounds, respectively:

$$
\begin{aligned}
& \text { MPE-DMPF } \\
& \text { MPE-DMPF }=I_{\mathrm{MPE}}^{\text {lower }}+D_{M}^{\text {lower }}, \\
& =I_{\mathrm{MPE}}^{\text {upper }}+D_{M}^{\text {upper }},
\end{aligned}
$$

where $I_{\mathrm{MPE}}^{\text {lower }}$ and $I_{\mathrm{MPE}}^{\text {upper }}$ represent the variation of the data in clusters of the dataset for the upper and lower bounds of the interval of clusters, respectively, and are represented by the following equations:

$$
\begin{aligned}
& I_{\mathrm{MPE}}^{\text {lower }}=-\frac{1}{n} \sum_{i=1}^{c} \sum_{k=1}^{n} \underline{u}_{i j}^{2} \log _{2} \underline{u}_{i j}, \\
& I_{\mathrm{MPE}}^{\mathrm{upper}}=-\frac{1}{n} \sum_{i=1}^{c} \sum_{k=1}^{n} \bar{u}_{i j}^{2} \log _{2} \bar{u}_{i j}
\end{aligned}
$$

and where $D_{M}^{\text {lower }}$ and $D_{M}^{\text {upper }}$ represent the separation between clusters in the dataset for the upper and lower bounds of the interval of clusters, respectively, and are represented by the following equations:

$$
\begin{aligned}
& D_{M_{k}}^{\text {lower }}=\sum_{\substack{i, j=1 \\
i \neq j}}^{k}\left\|M_{i}^{\text {lower }}-M_{j}^{\text {lower }}\right\|^{2}, \quad k=1, \ldots, c, \\
& D_{M_{k}}^{\text {upper }}=\sum_{\substack{i, j=1 \\
i \neq j}}^{k}\left\|M_{i}^{\text {upper }}-M_{j}^{\text {upper }}\right\|^{2}, \quad k=1, \ldots, c .
\end{aligned}
$$

\section{Results of the Implementation of the IT2FPCM Algorithm}

The IT2FPCM algorithm was tested with several benchmark datasets and images, in order to observe if the IT2FPCM algorithm is better than the IT2FCM algorithm. We perform 30 experiments using the Wine, WDBC, Iris Flower, Ionosphere, Abalone, and Cover type datasets. In order to observe the performance of the IT2FPCM algorithm against the IT2FCM algorithm we perform the data clustering of the datasets mentioned above with both algorithms mentioned above to compare the results obtained by these algorithms, and to measure the performance of these algorithms we use the validation indices mentioned in the previous section.

In Tables 1, 2, and 3, we show the results obtained for the WDBC dataset with 30 dimensions and 2 clusters with 569 samples; this dataset was tested with 2 to 10 clusters with the IT2FPCM and IT2FCM algorithms using different validation indices to evaluate the performance of both algorithms. The results that are shown are the mean of 30 experiments for each number of clusters tested in both algorithms. We can observe in Tables 1 and 2 that both algorithms find the correct 
TABLE 1: Results of the IT2MPE-DMFP validation index for the WDBC dataset clustering using IT2FPCM and IT2FCM algorithm using $m$ $=[1.5,2]$ and $\eta=[1.5,2.5]$ as the parameters.

\begin{tabular}{|c|c|c|c|c|c|c|c|}
\hline \multicolumn{8}{|c|}{ Index of validation IT2MPE-DMFP } \\
\hline \multirow{2}{*}{ Dataset } & \multirow{2}{*}{ Clusters } & \multicolumn{3}{|c|}{ IT2FPCM } & \multicolumn{3}{|c|}{ IT2FCM } \\
\hline & & Defuzz & Lower & Upper & Defuzz & Lower & Upper \\
\hline \multirow{9}{*}{ WDBC } & 2 & 0.59497 & 0.60327 & 0.58667 & 0.59374 & 0.60213 & 0.58536 \\
\hline & 3 & 1.38830 & 1.37432 & 1.40228 & 1.36790 & 1.35511 & 1.38069 \\
\hline & 4 & 1.57493 & 1.49703 & 1.65282 & 1.57353 & 1.49646 & 1.65060 \\
\hline & 5 & 2.02818 & 1.90438 & 2.15198 & 2.02736 & 1.90424 & 2.15048 \\
\hline & 6 & 2.68035 & 2.48420 & 2.87650 & 2.66823 & 2.47214 & 2.86432 \\
\hline & 7 & 2.85760 & 2.62283 & 3.09237 & 2.84878 & 2.61268 & 3.08488 \\
\hline & 8 & 3.81363 & 3.46430 & 4.16295 & 3.80316 & 3.45317 & 4.15314 \\
\hline & 9 & 4.63064 & 4.18376 & 5.07752 & 4.61201 & 4.16408 & 5.05994 \\
\hline & 10 & 4.80744 & 4.30470 & 5.31017 & 4.75902 & 4.25342 & 5.26462 \\
\hline
\end{tabular}

TABLE 2: Results of the IT2PE validation index for WDBC dataset clustering using IT2FPCM and IT2FCM algorithms with $m=[1.5,2]$ and $\eta=[1.5,2.5]$ as parameters.

\begin{tabular}{|c|c|c|c|c|c|c|c|}
\hline \multicolumn{8}{|c|}{ Index validation IT2PE } \\
\hline \multirow{2}{*}{ Dataset } & \multirow{2}{*}{ Clusters } & \multicolumn{3}{|c|}{ IT2FPCM } & \multicolumn{3}{|c|}{ IT2FCM } \\
\hline & & Defuzz & Lower & Upper & Defuzz & Lower & Upper \\
\hline \multirow{9}{*}{ WDBC } & 2 & 0.12504 & 0.10470 & 0.14539 & 0.12531 & 0.10476 & 0.14585 \\
\hline & 3 & 0.19601 & 0.15366 & 0.23835 & 0.20312 & 0.16011 & 0.24613 \\
\hline & 4 & 0.29863 & 0.24209 & 0.35517 & 0.29892 & 0.24215 & 0.35569 \\
\hline & 5 & 0.34488 & 0.26872 & 0.42105 & 0.34477 & 0.26853 & 0.42100 \\
\hline & 6 & 0.39213 & 0.30051 & 0.48375 & 0.39355 & 0.30176 & 0.48533 \\
\hline & 7 & 0.42646 & 0.32156 & 0.53137 & 0.42798 & 0.32287 & 0.53310 \\
\hline & 8 & 0.43077 & 0.32277 & 0.53877 & 0.43215 & 0.32387 & 0.54044 \\
\hline & 9 & 0.45241 & 0.33526 & 0.56956 & 0.45486 & 0.33697 & 0.57276 \\
\hline & 10 & 0.48363 & 0.35382 & 0.61344 & 0.48387 & 0.35353 & 0.61421 \\
\hline
\end{tabular}

TABLE 3: Results of the IT2XB validation index for WDBC dataset clustering using IT2FPCM and IT2FCM algorithm with $m=[1.5,2]$ and $\eta=[1.5,2.5]$ as parameters.

\begin{tabular}{|c|c|c|c|c|c|c|c|}
\hline \multicolumn{8}{|c|}{ Index validation IT2XB } \\
\hline \multirow{2}{*}{ Dataset } & \multirow{2}{*}{ Clusters } & \multicolumn{3}{|c|}{ IT2FPCM } & \multicolumn{3}{|c|}{ IT2FCM } \\
\hline & & Defuzz & Lower & Upper & Defuzz & Lower & Upper \\
\hline \multirow{9}{*}{ WDBC } & 2 & 0.06048 & 0.05100 & 0.06995 & 0.06137 & 0.05115 & 0.07159 \\
\hline & 3 & 0.06220 & 0.05061 & 0.07378 & 0.06586 & 0.05283 & 0.07889 \\
\hline & 4 & 0.17967 & 0.13465 & 0.22470 & 0.18209 & 0.13502 & 0.22915 \\
\hline & 5 & 0.18247 & 0.13453 & 0.23042 & 0.18533 & 0.13494 & 0.23572 \\
\hline & 6 & 0.17079 & 0.12100 & 0.22059 & 0.17506 & 0.12244 & 0.22768 \\
\hline & 7 & 0.22981 & 0.15569 & 0.30393 & 0.23242 & 0.15547 & 0.30937 \\
\hline & 8 & 0.19547 & 0.13585 & 0.25509 & 0.19844 & 0.13601 & 0.26088 \\
\hline & 9 & 0.17247 & 0.12025 & 0.22468 & 0.17563 & 0.12098 & 0.23028 \\
\hline & 10 & 0.18893 & 0.12504 & 0.25282 & 0.19963 & 0.13171 & 0.26754 \\
\hline
\end{tabular}

number of clusters for the lower and upper bound of the interval and its defuzzification using the IT2PE and IT2MPEDMFP validation indices. In Table 3 we can observe that with the IT2XB validation index the IT2FPCM did not find the correct number of clusters for the lower bound of the interval, but for the upper bound and defuzzification of the lower and upper bound of the interval it found the correct number of clusters.

In order to observe if there exists significant difference between the IT2FPCM and IT2FCM algorithms we perform a statistical test with the results obtained with the 3 validation indices for the results obtained by the clustering algorithms 
TABLE 4: Hypothesis testing for the IT2PE, IT2XB, and IT2MPE-DMFP indices of validation for the WDBC dataset clustering.

\begin{tabular}{ccccccccc}
\hline Dataset & Validation index & Algorithm & $N$ & $\mu$ & $\sigma^{2}$ & $z$-value & $z$-critical value & $P$ value \\
\hline \multirow{6}{*}{ WDBC } & \multirow{2}{*}{ IT2PE } & IT2FCM & \multirow{2}{*}{30} & 0.125307301 & $9.45 E-30$ & $-3.35094 E+11$ & 1.645 \\
& & IT2FPCM & & 0.125044778 & $8.96 E-30$ & & 0 \\
& IT2XB & IT2FCM & \multirow{2}{*}{30} & 0.061368738 & $1.75 E-29$ & $-8.46459 E+11$ & 1.645 \\
& & IT2FPCM & & 0.060476715 & $1.58 E-29$ & & 1.645 \\
& \multirow{2}{*}{ IT2MPE-DMFP } & IT2FCM & \multirow{2}{*}{30} & 0.593744494 & $1.06 E-27$ & $1.45455 E+11$ & 1 \\
& & IT2FPCM & & 0.594972091 & $1.08 E-27$ & & \\
\hline
\end{tabular}

TABLE 5: Results of the IT2MPE-DMFP validation index to Wine dataset clustering using IT2FPCM and IT2FCM algorithm using $m=[1.5$, 2] and $\eta=[1.5,2.5]$ as parameters.

\begin{tabular}{|c|c|c|c|c|c|c|c|}
\hline \multicolumn{8}{|c|}{ Index of validation IT2MPE-DMFP } \\
\hline \multirow{2}{*}{ Dataset } & \multirow{2}{*}{ Clusters } & \multicolumn{3}{|c|}{ IT2FPCM } & \multicolumn{3}{|c|}{ IT2FCM } \\
\hline & & Defuzz & Lower & Upper & Defuzz & Lower & Upper \\
\hline \multirow{9}{*}{ Wine } & 2 & 0.40744 & 0.41801 & 0.39686 & 0.40619 & 0.41684 & 0.39554 \\
\hline & 3 & 0.40225 & 0.39940 & 0.40509 & 0.40445 & 0.40146 & 0.40744 \\
\hline & 4 & 0.89656 & 0.87046 & 0.92267 & 0.89553 & 0.86964 & 0.92141 \\
\hline & 5 & 1.08796 & 1.03901 & 1.13691 & 1.08502 & 1.03721 & 1.13282 \\
\hline & 6 & 1.67907 & 1.51748 & 1.84066 & 1.90199 & 1.75824 & 2.04574 \\
\hline & 7 & 1.84502 & 1.67461 & 2.01543 & 2.86659 & 2.63321 & 3.09998 \\
\hline & 8 & 2.81205 & 2.54975 & 3.07434 & 1.68624 & 1.47369 & 1.89880 \\
\hline & 9 & 3.47078 & 3.14652 & 3.79504 & 3.46131 & 3.13748 & 3.78513 \\
\hline & 10 & 3.30712 & 2.92591 & 3.68834 & 3.30032 & 2.92011 & 3.68053 \\
\hline
\end{tabular}

mentioned above. The $z$-test was used with the following hypothesis for each validation index:

$$
\begin{aligned}
& H_{0} ; \text { IT2FPCM } \geq \text { IT2FCM } \\
& H_{1} ; \text { IT2FPCM < IT2FCM. }
\end{aligned}
$$

The hypothesis testing is performed for the best number of clusters found by the mentioned clustering algorithms. Table 4 shows the results from the hypothesis testing realized for the defuzzification of Type- 2 clusters of the WDBC dataset.

According to the assumptions made in (15), which arise in order to demonstrate that IT2FPCM algorithm is better than IT2FCM algorithm, in Table 4 we can observe the results of the $z$-test performed to data clustering of the WDBC dataset using the indices of validation mentioned in Section 4. In this case we can observe that the $z$-values of the hypothesis testing for the IT2PE, IT2XB, and IT2MPE-DMFP indices of validation are $-3.35094 E+11,-8.46459 E+11$, and $1.45455 E+11$, respectively. We can observe that the $z$-test shows that the IT2PE and IT2XB indices of validation are lower than the $z$-critical value that is equal to -1.645 with a significance level $\alpha$ of 0.05 , whose $z$-values confirm the acceptance of the alternative hypothesis posed in (15) for these indices of validation. In this way we demonstrate that the IT2FPCM algorithm is better than IT2FPCM algorithm for the data clustering of the WDBC dataset using the IT2PE and IT2XB indices. The $z$-value for hypothesis testing with the IT2MPE-DMFP index is greater than the $z$-critical value that is equal to -1.645 with a significance level $\alpha$ of 0.05 , whose $z$-value rejects the alternative hypothesis and accepts the null hypothesis posed in (15), demonstrating that there is no significant difference between the IT2FCM and IT2FPCM algorithms used in the $z$-test of the defuzzification.

In Tables 5, 6, and 7 we show the results obtained for the Wine dataset with 13 dimensions and 3 classes with 178 samples, and this dataset was tested with 2 to 10 clusters with the IT2FPCM and IT2FCM algorithms using different validation index to evaluate the performance of both algorithms. The results that are presented are the means of 30 experiments for each number of clusters used to test both algorithms; we can observe in Tables 8 and 9 that both algorithms did not find the correct number of clusters for the lower and upper bound of the interval and its defuzzification using the IT2PE and IT2XB validation index. In Table 7 we can observe that with the IT2MPE-DMFP validation index the IT2FPCM did not find the correct number of clusters for the upper bound of the interval, but to the lower bound and defuzzification of the lower and upper bound of the interval it did find the correct number of clusters.

According to the assumptions made in (15), which arise in order to demonstrate that the IT2FPCM algorithm is better than IT2FCM algorithm, in Table 8 we can observe the results of the $z$-test performed for data clustering of the Wine dataset using the indices of validation mentioned in Section 4. In this case we can observe that the $z$-values of the hypothesis testing for the IT2PE, IT2XB, and IT2MPE-DMFP indices of validation are $-1.01952 E+11,-7.83335 E+11$, and -22812613207 , respectively. We can notice that these values are lower than the $z$-critical value that is equal to -1.645 with a significant level $\alpha$ of 0.05 , and these $z$-values confirm the acceptance of the alternative hypothesis posed in (15) for 
TABLE 6: Results of the IT2PE validation index to Wine dataset clustering using IT2FPCM and IT2FCM algorithm with $m=[1.5,2]$ and $\eta=$ $[1.5,2.5]$ as parameters.

\begin{tabular}{|c|c|c|c|c|c|c|c|}
\hline \multicolumn{8}{|c|}{ Index of validation IT2PE } \\
\hline \multirow{2}{*}{ Dataset } & \multirow{2}{*}{ Clusters } & \multicolumn{3}{|c|}{ IT2FPCM } & \multicolumn{3}{|c|}{ IT2FCM } \\
\hline & & Defuzz & Lower & Upper & Defuzz & Lower & Upper \\
\hline \multirow{9}{*}{ Wine } & 2 & 0.14977 & 0.12509 & 0.17446 & 0.15006 & 0.12522 & 0.17491 \\
\hline & 3 & 0.26280 & 0.21554 & 0.31007 & 0.26287 & 0.21547 & 0.31028 \\
\hline & 4 & 0.28775 & 0.22242 & 0.35308 & 0.28769 & 0.22226 & 0.35311 \\
\hline & 5 & 0.33984 & 0.25551 & 0.42417 & 0.34005 & 0.25549 & 0.42461 \\
\hline & 6 & 0.36657 & 0.27738 & 0.45575 & 0.34110 & 0.25173 & 0.43048 \\
\hline & 7 & 0.33464 & 0.24323 & 0.42605 & 0.35228 & 0.25489 & 0.44966 \\
\hline & 8 & 0.34198 & 0.24496 & 0.43900 & 0.37465 & 0.26445 & 0.48484 \\
\hline & 9 & 0.36397 & 0.25674 & 0.47120 & 0.36531 & 0.25761 & 0.47300 \\
\hline & 10 & 0.39186 & 0.27080 & 0.51292 & 0.39213 & 0.27098 & 0.51328 \\
\hline
\end{tabular}

TABLE 7: Results of the IT2XB validation index to Wine dataset clustering using IT2FPCM and IT2FCM algorithm with $m=[1.5,2]$ and $\eta=$ $[1.5,2.5]$ as parameters.

\begin{tabular}{|c|c|c|c|c|c|c|c|}
\hline \multicolumn{8}{|c|}{ Index of validation IT2XB } \\
\hline \multirow{2}{*}{ Dataset } & \multirow{2}{*}{ Clusters } & \multicolumn{3}{|c|}{ IT2FPCM } & \multicolumn{3}{|c|}{ IT2FCM } \\
\hline & & Defuzz & Lower & Upper & Defuzz & Lower & Upper \\
\hline \multirow{9}{*}{ Wine } & 2 & 0.06009 & 0.05289 & 0.06730 & 0.06097 & 0.05301 & 0.06893 \\
\hline & 3 & 0.13492 & 0.10564 & 0.16421 & 0.13602 & 0.10573 & 0.16631 \\
\hline & 4 & 0.09865 & 0.07860 & 0.11871 & 0.09966 & 0.07883 & 0.12049 \\
\hline & 5 & 0.10894 & 0.08126 & 0.13661 & 0.10966 & 0.08169 & 0.13762 \\
\hline & 6 & 0.10862 & 0.08197 & 0.13526 & 0.08022 & 0.06075 & 0.09969 \\
\hline & 7 & 0.09032 & 0.06714 & 0.11350 & 0.08072 & 0.05840 & 0.10304 \\
\hline & 8 & 0.08295 & 0.06177 & 0.10414 & 0.12357 & 0.09168 & 0.15546 \\
\hline & 9 & 0.11040 & 0.08227 & 0.13852 & 0.11182 & 0.08301 & 0.14062 \\
\hline & 10 & 0.09347 & 0.06982 & 0.11712 & 0.09389 & 0.06992 & 0.11786 \\
\hline
\end{tabular}

TABLE 8: Hypothesis test for IT2PE, IT2XB, and IT2MPE-DMFP indices of validation for the Wine dataset clustering.

\begin{tabular}{|c|c|c|c|c|c|c|c|c|}
\hline Dataset & Validation Index & Algorithm & $N$ & $\mu$ & $\sigma^{2}$ & $z$-value & $z$-critical value & $P$ value \\
\hline \multirow{6}{*}{ Wine } & \multirow{2}{*}{ IT2PE } & IT2FCM & \multirow{2}{*}{30} & 0.150064427 & $1.14 E-28$ & \multirow{2}{*}{$-1.01952 E+11$} & \multirow{2}{*}{1.645} & \multirow{2}{*}{0} \\
\hline & & IT2FPCM & & 0.149773266 & $1.31 E-28$ & & & \\
\hline & \multirow{2}{*}{ IT2XB } & IT2FCM & \multirow{2}{*}{30} & 0.060970001 & $1.84 E-29$ & \multirow{2}{*}{$-7.83335 E+11$} & \multirow{2}{*}{1.645} & \multirow{2}{*}{0} \\
\hline & & IT2FPCM & & 0.060093276 & $1.92 E-29$ & & & \\
\hline & \multirow{2}{*}{ IT2MPE-DMFP } & IT2FCM & \multirow{2}{*}{30} & 0.404445222 & $1.37 E-25$ & \multirow[t]{2}{*}{-22812613207} & \multirow{2}{*}{1.645} & \multirow{2}{*}{0} \\
\hline & & IT2FPCM & & 0.402246542 & $1.42 E-25$ & & & \\
\hline
\end{tabular}

TABLE 9: Results of the IT2MPE-DMFP validation index to Iris Flower dataset clustering using IT2FPCM and IT2FCM algorithm with $m=$ $[1.5,2]$ and $\eta=[1.5,2.5]$ as parameters.

\begin{tabular}{|c|c|c|c|c|c|c|c|}
\hline \multicolumn{8}{|c|}{ Index validation IT2MPE-DMFP } \\
\hline \multirow{2}{*}{ Dataset } & \multirow{2}{*}{ Clusters } & \multicolumn{3}{|c|}{ IT2FPCM } & \multicolumn{3}{|c|}{ IT2FCM } \\
\hline & & Defuzz & Lower & Upper & Defuzz & Lower & Upper \\
\hline \multirow{9}{*}{ Iris } & 2 & 0.29224 & 0.30348 & 0.28101 & 0.29206 & 0.30341 & 0.28071 \\
\hline & 3 & 0.27581 & 0.27769 & 0.27394 & 0.27186 & 0.27371 & 0.27001 \\
\hline & 4 & 0.65772 & 0.70196 & 0.61348 & 0.78895 & 0.75105 & 0.82686 \\
\hline & 5 & 0.65979 & 0.59070 & 0.72888 & 0.64700 & 0.57492 & 0.71908 \\
\hline & 6 & 0.92039 & 0.79450 & 1.04628 & 1.66071 & 1.62370 & 1.69772 \\
\hline & 7 & 1.51645 & 1.31241 & 1.72049 & 0.85814 & 0.89191 & 0.82436 \\
\hline & 8 & 2.45753 & 2.32286 & 2.59220 & 1.41122 & 1.36998 & 1.45246 \\
\hline & 9 & 2.25261 & 2.08606 & 2.41917 & 1.90568 & 1.78587 & 2.02548 \\
\hline & 10 & 2.62804 & 2.40631 & 2.84977 & 2.60432 & 2.38812 & 2.82051 \\
\hline
\end{tabular}


TABLE 10: Results of the IT2PE validation index to Iris Flower dataset clustering using IT2FPCM and IT2FCM algorithm with $m=[1.5,2]$ and $\eta=[1.5,2.5]$ as parameters.

\begin{tabular}{|c|c|c|c|c|c|c|c|}
\hline \multirow{2}{*}{ Dataset } & \multirow{2}{*}{ Clusters } & \multicolumn{3}{|c|}{ IT2FPCM } & \multicolumn{3}{|c|}{ IT2FCM } \\
\hline & & Defuzz & Lower & Upper & Defuzz & Lower & Upper \\
\hline \multirow{9}{*}{ Iris } & 2 & 0.12706 & 0.10034 & 0.15378 & 0.12776 & 0.10080 & 0.15472 \\
\hline & 3 & 0.27001 & 0.21982 & 0.32020 & 0.27104 & 0.22074 & 0.32134 \\
\hline & 4 & 0.38333 & 0.30413 & 0.46252 & 0.38442 & 0.30513 & 0.46370 \\
\hline & 5 & 0.45027 & 0.34297 & 0.55758 & 0.45232 & 0.34465 & 0.55999 \\
\hline & 6 & 0.54534 & 0.42762 & 0.66305 & 0.54757 & 0.42954 & 0.66560 \\
\hline & 7 & 0.63092 & 0.49076 & 0.77108 & 0.63321 & 0.49262 & 0.77379 \\
\hline & 8 & 0.67371 & 0.51134 & 0.83607 & 0.68029 & 0.51647 & 0.84411 \\
\hline & 9 & 0.74321 & 0.56287 & 0.92354 & 0.73528 & 0.53285 & 0.93771 \\
\hline & 10 & 0.80024 & 0.60567 & 0.99480 & 0.80718 & 0.61146 & 1.00291 \\
\hline
\end{tabular}

TABLE 11: Results of the IT2XB validation index to Iris Flower dataset clustering using IT2FPCM and IT2FCM algorithm with $m=[1.5,2]$ and $\eta=[1.5,2.5]$ as parameters.

\begin{tabular}{|c|c|c|c|c|c|c|c|}
\hline \multirow{2}{*}{ Dataset } & \multirow{2}{*}{ Clusters } & \multicolumn{3}{|c|}{ IT2FPCM } & \multicolumn{3}{|c|}{ IT2FCM } \\
\hline & & Defuzz & Lower & Upper & Defuzz & Lower & Upper \\
\hline \multirow{9}{*}{ Iris } & 2 & 0.05604 & 0.04877 & 0.06331 & 0.05656 & 0.04870 & 0.06442 \\
\hline & 3 & 0.13410 & 0.11048 & 0.15772 & 0.13778 & 0.11084 & 0.16472 \\
\hline & 4 & 0.18766 & 0.14543 & 0.22990 & 0.19211 & 0.14687 & 0.23736 \\
\hline & 5 & 0.22753 & 0.16904 & 0.28602 & 0.23302 & 0.17094 & 0.29509 \\
\hline & 6 & 0.29483 & 0.21162 & 0.37804 & 0.30525 & 0.21204 & 0.39846 \\
\hline & 7 & 0.31905 & 0.23313 & 0.40497 & 0.33896 & 0.23724 & 0.44067 \\
\hline & 8 & 0.23901 & 0.17431 & 0.30371 & 0.24926 & 0.17482 & 0.32369 \\
\hline & 9 & 0.39515 & 0.27792 & 0.51239 & 0.55028 & 0.38453 & 0.71604 \\
\hline & 10 & 0.34780 & 0.23825 & 0.45735 & 0.35965 & 0.24133 & 0.47796 \\
\hline
\end{tabular}

TABLE 12: Hypothesis testing for the IT2PE, IT2XB, and IT2MPE-DMFP indices of validation for the Iris Flower dataset clustering.

\begin{tabular}{lcccccccc}
\hline Dataset & Validation index & Algorithm & $N$ & $\mu$ & $\sigma^{2}$ & $z$-value & $z$-critical value & $P$ value \\
\hline \multirow{5}{*}{ Iris Flower } & \multirow{2}{*}{ IT2PE } & IT2FCM & \multirow{2}{*}{30} & 0.127758962 & $2.81 E-24$ & -1447853758 & 1.645 \\
& & IT2FPCM & & 0.127055881 & $4.27 E-24$ & & 0 \\
& IT2XB & IT2FCM & \multirow{2}{*}{30} & 0.056558597 & $9.64 E-28$ & -38476101768 & 1.645 \\
& & IT2FPCM & & 0.056038216 & $4.52 E-27$ & & 0 \\
& & IT2FCM & \multirow{2}{*}{30} & 0.271858146 & $5.91 E-21$ & 184969393.7 & 1.645 \\
\hline
\end{tabular}

all the tested indices of validation, demonstrating that the IT2FPCM algorithm is better than the IT2FPCM algorithm for the data clustering of the Wine dataset. In Tables 9, 10, and 11 we show the results obtained for a Iris Flower dataset with 4 dimensions and 3 classes with 150 samples, and this dataset was tested with 2 to 10 clusters with the IT2FPCM and IT2FCM algorithms using different validation indices to evaluate the performance of both algorithms and the results shown are the mean of 30 experiments for each number of clusters used tested in both algorithms. In Table 9 we can observe that both algorithms with the IT2MPE-DMFP index validation did find the correct number of clusters for the lower and upper bounds of the limit and its defuzzification. On the other hand, in Tables 10 and 11 the IT2PE and IT2XB algorithms did not find the correct number the clusters.
In Table 12 we can observe the results of the $z$-test performed to the data clustering of the Iris Flower dataset using the indices of validation mentioned in Section 4. In this case we can observe that the $z$-values of the hypothesis testing for the IT2PE, IT2XB, and IT2MPE-DMFP indices of validation are $-1447853758,-38476101768$, and 184969393.7, respectively. We can observe that $z$-test shows that the IT2PE and IT2XB indices of validation are lower than the $z$-critical value that is equal to -1.645 with a significance level $\alpha$ of 0.05 , whose $z$-values confirm the acceptance of the alternative hypothesis posed in (15) for these indices of validation, demonstrating that the IT2FPCM algorithm is better than IT2FPCM algorithm for the data clustering of the Iris Flower dataset using the IT2PE and IT2XB indices. However, the $z$-value for the test with the IT2MPE-DMFP index is greater than the $z$-critical value that is equal to -1.645 
TABLE 13: IT2MPE-DMFP validation index results to Ionosphere dataset clustering using IT2FPCM and IT2FCM algorithm with $m=[1.5$, $2.5]$ and $\eta=[1.5,2.5]$ as parameters.

\begin{tabular}{|c|c|c|c|c|c|c|c|}
\hline \multicolumn{8}{|c|}{ Index of validation IT2MPEDFP } \\
\hline \multirow{2}{*}{ Dataset } & \multirow{2}{*}{ Clusters } & \multicolumn{3}{|c|}{ IT2FPCM } & \multicolumn{3}{|c|}{ IT2FCM } \\
\hline & & Defuzz & Lower & Upper & Defuzz & Lower & Upper \\
\hline \multirow{9}{*}{ Ionosphere } & 2 & 0.37490 & 0.38189 & 0.36792 & 0.37872 & 0.38264 & 0.37479 \\
\hline & 3 & 0.67283 & 0.57794 & 0.76772 & 0.67487 & 0.57848 & 0.77126 \\
\hline & 4 & 0.83519 & 0.69115 & 0.97923 & 0.83839 & 0.69166 & 0.98511 \\
\hline & 5 & 1.01579 & 0.80954 & 1.22204 & 1.01694 & 0.80933 & 1.22456 \\
\hline & 6 & 1.17398 & 0.89175 & 1.45622 & 1.17490 & 0.89191 & 1.45789 \\
\hline & 7 & 1.31217 & 0.95649 & 1.66785 & 1.31246 & 0.95366 & 1.67126 \\
\hline & 8 & 1.47017 & 1.03109 & 1.90924 & 1.46777 & 1.02669 & 1.90885 \\
\hline & 9 & 1.64906 & 1.09276 & 2.20535 & 1.64527 & 1.08737 & 2.20317 \\
\hline & 10 & 1.79078 & 1.15390 & 2.42766 & 1.78653 & 1.14450 & 2.42856 \\
\hline
\end{tabular}

TABLE 14: Results of the IT2PE validation index to Ionosphere dataset clustering using IT2FPCM and IT2FCM algorithm with $m=[1.5,2.5]$ and $\eta=[1.5,2.5]$ as parameters.

\begin{tabular}{|c|c|c|c|c|c|c|c|}
\hline \multicolumn{8}{|c|}{ Index of validation IT2PE } \\
\hline \multirow{2}{*}{ Dataset } & \multirow{2}{*}{ Clusters } & \multicolumn{3}{|c|}{ IT2FPCM } & \multicolumn{3}{|c|}{ IT2FCM } \\
\hline & & Defuzz & Lower & Upper & Defuzz & Lower & Upper \\
\hline \multirow{9}{*}{ Ionosphere } & 2 & 0.46246 & 0.46354 & 0.46138 & 0.46629 & 0.46478 & 0.46780 \\
\hline & 3 & 0.80424 & 0.75328 & 0.85520 & 0.80622 & 0.75262 & 0.85982 \\
\hline & 4 & 1.01893 & 0.92719 & 1.11067 & 1.02047 & 0.92553 & 1.11541 \\
\hline & 5 & 1.20877 & 1.07092 & 1.34663 & 1.20802 & 1.06779 & 1.34826 \\
\hline & 6 & 1.37426 & 1.20016 & 1.54836 & 1.37065 & 1.19522 & 1.54607 \\
\hline & 7 & 1.52065 & 1.31404 & 1.72727 & 1.51381 & 1.30717 & 1.72044 \\
\hline & 8 & 1.65658 & 1.41618 & 1.89699 & 1.64516 & 1.40690 & 1.88342 \\
\hline & 9 & 1.77473 & 1.50879 & 2.04066 & 1.75803 & 1.49612 & 2.01995 \\
\hline & 10 & 1.87674 & 1.58679 & 2.16670 & 1.85595 & 1.57139 & 2.14051 \\
\hline
\end{tabular}

TABLE 15: Results of the IT2XB validation index to Ionosphere dataset clustering using IT2FPCM and IT2FCM algorithm with $m=[1.5,2.5]$ and $\eta=[1.5,2.5]$ as parameters.

\begin{tabular}{|c|c|c|c|c|c|c|c|}
\hline \multicolumn{8}{|c|}{ Index of validation IT2XB } \\
\hline \multirow{2}{*}{ Dataset } & \multirow{2}{*}{ Clusters } & \multicolumn{3}{|c|}{ IT2FPCM } & \multicolumn{3}{|c|}{ IT2FCM } \\
\hline & & Defuzz & Lower & Upper & Defuzz & Lower & Upper \\
\hline \multirow{9}{*}{ Ionosphere } & 2 & 0.62505 & 0.51872 & 0.73137 & 0.62236 & 0.51799 & 0.72674 \\
\hline & 3 & 2.36442 & 3.07170 & 1.65714 & 2.34434 & 3.05359 & 1.63509 \\
\hline & 4 & 1.25146 & 1.18415 & 1.31877 & 1.24377 & 1.18394 & 1.30359 \\
\hline & 5 & $4.05 E+04$ & $5.90 E+04$ & $2.20 E+04$ & $1.93 E+06$ & $2.82 E+06$ & $1.03 E+06$ \\
\hline & 6 & $5.58 E+03$ & $7.99 E+03$ & $3.17 E+03$ & $6.35 E+06$ & $9.12 E+06$ & $3.58 E+06$ \\
\hline & 7 & $2.46 E+11$ & $3.52 E+11$ & $1.39 E+11$ & $5.22 E+11$ & $7.51 E+11$ & $2.92 E+11$ \\
\hline & 8 & $1.98 E+15$ & $2.74 E+15$ & $1.23 E+15$ & $3.38 E+16$ & $4.71 E+16$ & $2.05 E+16$ \\
\hline & 9 & $1.69 E+14$ & $2.29 E+14$ & $1.08 E+14$ & $1.70 E+14$ & $2.32 E+14$ & $1.08 E+14$ \\
\hline & 10 & $6.22 E+20$ & $8.71 E+20$ & $3.73 E+20$ & $1.31 E+21$ & $1.85 E+21$ & $7.62 E+20$ \\
\hline
\end{tabular}

with a significance level $\alpha$ of 0.05 , whose $z$-value rejects the alternative hypothesis and accepts the null hypothesis posed in (15), demonstrating that there is no significant difference between IT2FCM and IT2FPCM algorithms used for the $z$ test of the defuzzification.

In Tables 13, 14, and 15 we show the results obtained for the Ionosphere dataset with 34 dimensions and 2 classes with 351 samples. This dataset was tested with 2 to 10 clusters with the IT2FPCM and IT2FCM algorithms using different validation indices to evaluate the performance of both algorithms. The results presented are the means of 30 experiments for each number of clusters used in both algorithms. In Tables 13, 14, and 15 we can observe that both algorithms find the correct number of clusters for the Ionosphere dataset with all the 
TABLE 16: Hypothesis test for IT2PE, IT2XB, and IT2MPE-DMFP indices of validation for the Ionosphere dataset clustering.

\begin{tabular}{lcccccccc}
\hline Dataset & Validation index & Algorithm & $N$ & $\mu$ & $\sigma^{2}$ & $z$-value & $z$-critical value $P$ value \\
\hline \multirow{5}{*}{ Ionosphere } & \multirow{2}{*}{ IT2PE } & IT2FCM & \multirow{2}{*}{30} & 0.466290024 & $0.00 E+00$ & $-7.55279 E+13$ & 1.645 \\
& & IT2FPCM & & 0.462462683 & $7.70 E-32$ & & 0 \\
& \multirow{2}{*}{ IT2XB } & IT2FCM & \multirow{2}{*}{30} & 0.622362066 & $1.89 E-31$ & $2.66339 E+13$ & 1.645 \\
& IT2FPCM & & 0.625045136 & $1.16 E-31$ & & 1 \\
& \multirow{2}{*}{ IT2MPE-DMFP } & IT2FCM & \multirow{2}{*}{30} & 0.378715301 & $7.70 E-32$ & $-3.89081 E+13$ & 1.645 \\
& & IT2FPCM & & 0.374904357 & $2.11 E-31$ & & 0 \\
\hline
\end{tabular}

TABLE 17: IT2MPE-DMFP validation index results to Abalone dataset clustering using IT2FPCM and IT2FCM algorithm with $m=[1.5,2.5]$ and $\eta=[1.5,2.5]$ as parameters.

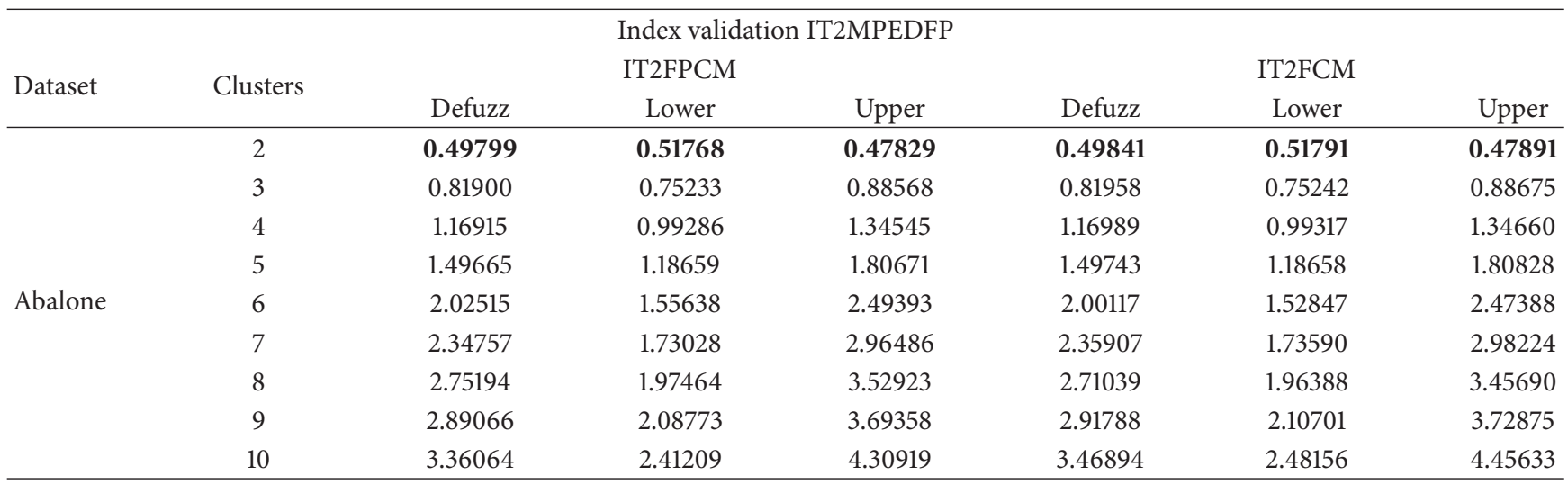

TABLE 18: Results of the IT2PE validation index to Abalone dataset clustering using IT2FPCM and IT2FCM algorithm with $m=[1.5,2.5]$ and $\eta=[1.5,2.5]$ as parameters.

\begin{tabular}{|c|c|c|c|c|c|c|c|}
\hline \multicolumn{8}{|c|}{ Index of validation IT2PE } \\
\hline \multirow{2}{*}{ Dataset } & \multirow{2}{*}{ Clusters } & \multicolumn{3}{|c|}{ IT2FPCM } & \multicolumn{3}{|c|}{ IT2FCM } \\
\hline & & Defuzz & Lower & Upper & Defuzz & Lower & Upper \\
\hline \multirow{9}{*}{ Abalone } & 2 & 0.28046 & 0.24561 & 0.31530 & 0.28088 & 0.24588 & 0.31587 \\
\hline & 3 & 0.41503 & 0.33056 & 0.49950 & 0.41555 & 0.33088 & 0.50022 \\
\hline & 4 & 0.51390 & 0.38126 & 0.64654 & 0.51451 & 0.38167 & 0.64735 \\
\hline & 5 & 0.58848 & 0.40296 & 0.77400 & 0.58910 & 0.40341 & 0.77479 \\
\hline & 6 & 0.65438 & 0.42798 & 0.88077 & 0.65645 & 0.42848 & 0.88441 \\
\hline & 7 & 0.72528 & 0.46266 & 0.98791 & 0.72530 & 0.46222 & 0.98837 \\
\hline & 8 & 0.77107 & 0.47984 & 1.06230 & 0.77356 & 0.48179 & 1.06533 \\
\hline & 9 & 0.79848 & 0.48202 & 1.11494 & 0.80140 & 0.48452 & 1.11828 \\
\hline & 10 & 0.81290 & 0.47804 & 1.14776 & 0.81251 & 0.47777 & 1.14725 \\
\hline
\end{tabular}

validation indices used to measure the performance of the both algorithms.

In Table 16 we can observe the results of the $z$-tests performed for the clustering of the Ionosphere dataset using the indices of validation mentioned in Section 4. In this case we can observe that the $z$-values of the hypothesis testing with the IT2PE, IT2XB, and IT2MPE-DMFP indices of validation are $-7.55279 E+13,2.66339 E+13$, and $-3.89081 E+13$, respectively. We can observe that $z$-test shows that the IT2PE and IT2MPE-DMFP indices of validation are lower than the $z$-critical value that is equal to -1.645 with a significance level $\alpha$ of 0.05 , and these $z$-values confirm the acceptance of the alternative hypothesis posed in (15) for these indices of validation, demonstrating that the IT2FPCM algorithm is better than the IT2FPCM algorithm for the data clustering of the Ionosphere dataset using the IT2PE and IT2MPE-DMFP indices. The $z$-value for the test with the IT2XB index shows that is greater than the $z$-critical value that is equal to -1.645 with a significance level $\alpha$ of 0.05 , and this $z$-value rejects the alternative hypothesis and accepts the null hypothesis posed in (15), demonstrating that there is no significant difference between the IT2FCM and IT2FPCM algorithms used in the $z$-test of the defuzzification for the IT2XB index.

In Tables 17, 18, and 19 we show the results obtained for the Abalone dataset with 8 dimensions and 3 classes according to the sex of the Abalone and with 4177 samples. This dataset was tested with 2 to 10 clusters with the IT2FPCM and IT2FCM algorithms using different validation index to 
TABLE 19: Results of the IT2XB validation index to Abalone dataset clustering using IT2FPCM and IT2FCM algorithm with $m=[1.5,2.5]$ and $\eta=[1.5,2.5]$ as parameters.

\begin{tabular}{|c|c|c|c|c|c|c|c|}
\hline \multicolumn{8}{|c|}{ Index of validation IT2XB } \\
\hline \multirow{2}{*}{ Dataset } & \multirow{2}{*}{ Clusters } & \multicolumn{3}{|c|}{ IT2FPCM } & \multicolumn{3}{|c|}{ IT2FCM } \\
\hline & & Defuzz & Lower & Upper & Defuzz & Lower & Upper \\
\hline \multirow{9}{*}{ Abalone } & 2 & 0.12162 & 0.08980 & 0.15344 & 0.12160 & 0.08980 & 0.15340 \\
\hline & 3 & 0.15659 & 0.08965 & 0.22353 & 0.15651 & 0.08964 & 0.22338 \\
\hline & 4 & 0.14604 & 0.06983 & 0.22224 & 0.14592 & 0.06982 & 0.22202 \\
\hline & 5 & 0.19353 & 0.08216 & 0.30489 & 0.19328 & 0.08212 & 0.30445 \\
\hline & 6 & 0.18472 & 0.07469 & 0.29474 & 0.18444 & 0.07514 & 0.29374 \\
\hline & 7 & 0.18783 & 0.07297 & 0.30269 & 0.18551 & 0.07230 & 0.29873 \\
\hline & 8 & 0.23117 & 0.08481 & 0.37753 & 0.23847 & 0.08663 & 0.39030 \\
\hline & 9 & 0.34175 & 0.11190 & 0.57161 & 0.34974 & 0.11459 & 0.58489 \\
\hline & 10 & 0.32883 & 0.10652 & 0.55115 & 0.32309 & 0.10437 & 0.54182 \\
\hline
\end{tabular}

TABLE 20: Hypothesis test for IT2PE, IT2XB, and IT2MPE-DMFP indices of validation for the Abalone dataset clustering.

\begin{tabular}{|c|c|c|c|c|c|c|c|c|}
\hline Dataset & Validation index & Algorithm & $N$ & $\mu$ & $\sigma^{2}$ & $z$-value & $z$-critical value & $P$ value \\
\hline \multirow{6}{*}{ Abalone } & \multirow{2}{*}{ IT2PE } & IT2FCM & \multirow{2}{*}{30} & 0.280876194 & $2.77 E-32$ & \multirow{2}{*}{$-1.30990 E+13$} & \multirow{2}{*}{1.645} & \multirow{2}{*}{0} \\
\hline & & IT2FPCM & & 0.280456378 & $3.08 E-33$ & & & \\
\hline & \multirow{2}{*}{ IT2XB } & IT2FCM & \multirow{2}{*}{30} & 0.121597213 & $3.08 E-33$ & \multirow{2}{*}{$1.78710 E+12$} & \multirow{2}{*}{1.645} & \multirow{2}{*}{1} \\
\hline & & IT2FPCM & & 0.121619853 & $1.73 E-33$ & & & \\
\hline & \multirow{2}{*}{ IT2MPE-DMFP } & IT2FCM & \multirow{2}{*}{30} & 0.498409184 & $9.18 E-32$ & \multirow{2}{*}{$-5.21128 E+12$} & \multirow{2}{*}{1.645} & \multirow[t]{2}{*}{0} \\
\hline & & IT2FPCM & & 0.497985339 & $1.07 E-31$ & & & \\
\hline
\end{tabular}

evaluate the performance of both algorithms. The results presented in the tables are the means of 30 experiments for each number of clusters used in both algorithms. In Tables 17,18 , and 19 we can observe that both algorithms fail to find the correct number of clusters for the Abalone dataset with all validation indices used to measure the performance of the both algorithms.

In Table 20 we can observe the results of the $z$-test performed to data clustering for the Abalone dataset using the indices of validation mentioned in Section 4, where we can observe that the $z$-values of the hypothesis test to the indices of validation IT2PE, IT2XB, and IT2MPE-DMFP are $-1.30990 E+13,1.78710 E+12$, and $-5.21128 E+12$, respectively. In this case we can observe that $z$-test shows that IT2PE and IT2MPE-DMFP indices of validation are lower than the $z$-critical value that is equal to -1.645 with a significance level $\alpha$ of 0.05 , whose $z$-values confirm the acceptance of the alternative hypothesis posed in (15) for these indices of validation, demonstrating that the IT2FPCM algorithm is better than the IT2FPCM algorithm for the data clustering of the Ionosphere dataset using the IT2PE and IT2MPE-DMFP indices; the $z$-value for the hypothesis test of the IT2XB index is greater than the $z$-critical value that is equal to -1.645 with a significant level $(\alpha)$ of 0.05 , whose $z$-value rejects the alternative hypothesis and accepts the null hypothesis posed in (15), demonstrating that there is no significant difference between IT2FCM and IT2FPCM algorithm used to $z$-test of the defuzzification for the IT2XB index.
In Tables 21, 22, and 23 we show the results obtained for a Cover type dataset with 54 dimensions and 7 classes with 581012 samples, and this dataset was tested with 2 to 9 clusters with the IT2FPCM and IT2FCM algorithm using different validation index to evaluate the performance of both algorithms. The results shown are the means of 30 experiments for each number of clusters used tested in both algorithms. In Tables 21, 22, and 23 we can observe that both algorithms fail in finding the correct number of clusters for the Cover type dataset with all validation index used to measure the performance of the both algorithms.

In Table 24 we can observe the results of the $z$-test performed for data clustering of the Cover type dataset using the indices of validation mentioned in Section 4, where we can observe that the $z$-values of the hypothesis testing for the IT2PE, IT2XB, and IT2MPE-DMFP indices of validation are $-8.15509 E+10,6.67332 E+02$, and $-3.12019 E+10$, respectively. In this case we can observe that the $z$-test shows that the IT2PE and IT2MPE-DMFP indices of validation are lower than the $z$-critical value that is equal to -1.645 with a significance level $\alpha$ of 0.05 , whose $z$-values confirm the acceptance of the alternative hypothesis posed in (15) for these indices of validation tested, demonstrating that the IT2FPCM algorithm is better than the IT2FPCM algorithm for the data clustering of the Ionosphere dataset using the IT2PE and IT2MPE-DMFP indices. The $z$-value for the hypothesis test for the IT2XB index is greater than the $z$ critical value that is equal to -1.645 with a significance level $\alpha$ of 0.05 , whose $z$-value rejects the alternative hypothesis and 
TABLE 21: IT2MPE-DMFP validation index results to Cover type dataset clustering using IT2FPCM and IT2FCM algorithm with $m=[1.5$, $2.5]$ and $\eta=[1.5,2.5]$ as parameters.

\begin{tabular}{|c|c|c|c|c|c|c|c|}
\hline \multicolumn{8}{|c|}{ Index of validation IT2MPEDFP } \\
\hline \multirow{2}{*}{ Dataset } & \multirow{2}{*}{ Clusters } & \multicolumn{3}{|c|}{ IT2FPCM } & \multicolumn{3}{|c|}{ IT2FCM } \\
\hline & & Defuzz & Lower & Upper & Defuzz & Lower & Upper \\
\hline \multirow{8}{*}{ Cover type } & 2 & 0.44077 & 0.45732 & 0.42422 & 0.44077 & 0.45732 & 0.42422 \\
\hline & 3 & 0.87629 & 0.82323 & 0.92935 & 0.87629 & 0.82323 & 0.92936 \\
\hline & 4 & 1.04019 & 0.91650 & 1.16388 & 1.04019 & 0.91650 & 1.16389 \\
\hline & 5 & 1.16102 & 0.94169 & 1.38036 & 1.16102 & 0.94168 & 1.38037 \\
\hline & 6 & 1.32155 & 1.01723 & 1.62587 & 1.32183 & 1.01743 & 1.62623 \\
\hline & 7 & 1.66573 & 1.24415 & 2.08731 & 1.67645 & 1.25221 & 2.10069 \\
\hline & 8 & 1.79403 & 1.30555 & 2.28251 & 1.78721 & 1.29820 & 2.27623 \\
\hline & 9 & 1.92141 & 1.34475 & 2.49807 & 1.93580 & 1.35667 & 2.51492 \\
\hline
\end{tabular}

TABLE 22: Results of the IT2PE validation index to Cover type dataset clustering using IT2FPCM and IT2FCM algorithm with $m=[1.5,2.5]$ and $\eta=[1.5,2.5]$ as parameters.

\begin{tabular}{|c|c|c|c|c|c|c|c|}
\hline \multirow{3}{*}{ Dataset } & \multirow{3}{*}{ Clusters } & \multicolumn{3}{|c|}{ Index of validation IT2PE } & \multirow{2}{*}{\multicolumn{3}{|c|}{ IT2FCM }} \\
\hline & & & IT2FPCN & & & & \\
\hline & & Defuzz & Lower & Upper & Defuzz & Lower & Upper \\
\hline \multirow{8}{*}{ Cover type } & 2 & 0.35587 & 0.33093 & 0.38080 & 0.35587 & 0.33093 & 0.38081 \\
\hline & 3 & 0.56607 & 0.47652 & 0.65563 & 0.56608 & 0.47652 & 0.65563 \\
\hline & 4 & 0.73183 & 0.58434 & 0.87933 & 0.73183 & 0.58434 & 0.87933 \\
\hline & 5 & 0.85155 & 0.64428 & 1.05882 & 0.85155 & 0.64428 & 1.05883 \\
\hline & 6 & 0.97154 & 0.70936 & 1.23372 & 0.97152 & 0.70934 & 1.23370 \\
\hline & 7 & 1.05830 & 0.75102 & 1.36559 & 1.05713 & 0.74991 & 1.36436 \\
\hline & 8 & 1.14555 & 0.79324 & 1.49786 & 1.14611 & 0.79369 & 1.49853 \\
\hline & 9 & 1.22450 & 0.83102 & 1.61797 & 1.22440 & 0.83113 & 1.61766 \\
\hline
\end{tabular}

TABLE 23: Results of the IT2XB validation index to Cover type dataset clustering using IT2FPCM and IT2FCM algorithm with $m=[1.5,2.5]$ and $\eta=[1.5,2.5]$ as parameters.

\begin{tabular}{|c|c|c|c|c|c|c|c|}
\hline \multicolumn{8}{|c|}{ Index of validation IT2XB } \\
\hline \multirow{2}{*}{ Dataset } & \multirow{2}{*}{ Clusters } & \multicolumn{3}{|c|}{ IT2FPCM } & \multicolumn{3}{|c|}{ IT2FCM } \\
\hline & & Defuzz & Lower & Upper & Defuzz & Lower & Upper \\
\hline \multirow{8}{*}{ Cover Type } & 2 & 0.19894 & 0.14137 & 0.25651 & 0.19894 & 0.14137 & 0.25651 \\
\hline & 3 & 0.19557 & 0.11641 & 0.27474 & 0.19557 & 0.11641 & 0.27474 \\
\hline & 4 & 0.33846 & 0.19325 & 0.48366 & 0.33845 & 0.19325 & 0.48366 \\
\hline & 5 & 0.24190 & 0.11492 & 0.36887 & 0.24189 & 0.11492 & 0.36886 \\
\hline & 6 & 0.24645 & 0.10625 & 0.38665 & 0.24628 & 0.10618 & 0.38639 \\
\hline & 7 & 0.29318 & 0.12373 & 0.46263 & 0.29165 & 0.12304 & 0.46026 \\
\hline & 8 & 0.34262 & 0.14107 & 0.54418 & 0.33522 & 0.13744 & 0.53301 \\
\hline & 9 & 0.28467 & 0.10933 & 0.46002 & 0.28741 & 0.11148 & 0.46335 \\
\hline
\end{tabular}

TABLE 24: Hypothesis test for IT2PE, IT2XB, and IT2MPE-DMFP indices of validation for the Abalone dataset clustering.

\begin{tabular}{ccccccccc}
\hline Dataset & Validation index & Algorithm & $N$ & $\mu$ & $\sigma^{2}$ & $z$-value & $z$-critical value $P$ value \\
& \multirow{6}{*}{ IT2PE } & IT2FCM & \multirow{2}{*}{30} & 0.355868292 & $2.77 E-32$ & $-8.15509 E+10$ & 1.645 \\
& & IT2FPCM & & 0.355865678 & $3.08 E-33$ & & 0 \\
\multirow{6}{*}{ Abalone } & IT2XB & IT2FCM & \multirow{2}{*}{30} & 0.195572668 & $5.33 E-17$ & $6.67332 E+02$ & 1.645 \\
& & IT2FPCM & & 0.195573745 & $2.49 E-17$ & & 1 \\
& \multirow{2}{*}{ IT2MPE-DMFP } & IT2FCM & \multirow{2}{*}{30} & 0.440773577 & $1.89 E-31$ & $-3.12019 E+10$ & 1.645 \\
& & IT2FPCM & & 0.440771083 & $3.08 E-33$ & & 0 \\
\hline
\end{tabular}


TABLE 25: Results of the IT2MPE-DMFP validation index to data clustering of image shown in Figure 2(a) using IT2FPCM and IT2FCM algorithm with $m=[1.5,2.5]$ and $\eta=[1.5,2.5]$ as parameters.

\begin{tabular}{|c|c|c|c|c|c|c|c|}
\hline \multicolumn{8}{|c|}{ Index of validation IT2MPE-DMFP } \\
\hline \multirow{2}{*}{ Image } & \multirow{2}{*}{ Clusters } & \multicolumn{3}{|c|}{ IT2FPCM } & \multicolumn{3}{|c|}{ IT2FCM } \\
\hline & & Defuzz & Lower & Upper & Defuzz & Lower & Upper \\
\hline \multirow{9}{*}{ Figure 2(a) } & 2 & 0.31330 & 0.32940 & 0.29720 & 0.31331 & 0.32941 & 0.29722 \\
\hline & 3 & 0.64736 & 0.61172 & 0.68301 & 0.64738 & 0.61172 & 0.68304 \\
\hline & 4 & 0.87847 & 0.78421 & 0.97273 & 0.87849 & 0.78421 & 0.97278 \\
\hline & 5 & 1.25053 & 1.08130 & 1.41975 & 1.25056 & 1.08131 & 1.41981 \\
\hline & 6 & 1.52447 & 1.27572 & 1.77322 & 1.52451 & 1.27573 & 1.77329 \\
\hline & 7 & 1.80474 & 1.48249 & 2.12700 & 1.80483 & 1.48250 & 2.12716 \\
\hline & 8 & 2.01639 & 1.61788 & 2.41490 & 2.01654 & 1.61781 & 2.41527 \\
\hline & 9 & 2.29257 & 1.81493 & 2.77020 & 2.29500 & 1.81652 & 2.77349 \\
\hline & 10 & 2.60279 & 2.03320 & 3.17238 & 2.60219 & 2.03318 & 3.17121 \\
\hline
\end{tabular}

TABLE 26: Results of the IT2PE validation index to the clustering of image shown in Figure 2(a) using IT2FPCM and IT2FCM algorithms with $m=[1.5,2.5]$ and $\eta=[1.5,2.5]$ as parameters.

\begin{tabular}{|c|c|c|c|c|c|c|c|}
\hline \multirow{3}{*}{ Image } & \multirow{3}{*}{ Clusters } & \multicolumn{3}{|c|}{ Index of validation IT2PE } & \multirow{2}{*}{\multicolumn{3}{|c|}{ IT2FCM }} \\
\hline & & & IT2FPCM & & & & \\
\hline & & Defuzz & Lower & Upper & Defuzz & Lower & Upper \\
\hline \multirow{9}{*}{ Figure 2(a) } & 2 & 0.27752 & 0.24869 & 0.30635 & 0.27753 & 0.24869 & 0.30637 \\
\hline & 3 & 0.38492 & 0.30127 & 0.46858 & 0.38494 & 0.30128 & 0.46861 \\
\hline & 4 & 0.47242 & 0.34265 & 0.60219 & 0.47244 & 0.34267 & 0.60222 \\
\hline & 5 & 0.52743 & 0.36020 & 0.69467 & 0.52746 & 0.36022 & 0.69470 \\
\hline & 6 & 0.56679 & 0.36941 & 0.76416 & 0.56682 & 0.36943 & 0.76421 \\
\hline & 7 & 0.60220 & 0.37799 & 0.82641 & 0.60223 & 0.37800 & 0.82646 \\
\hline & 8 & 0.63271 & 0.38504 & 0.88037 & 0.63277 & 0.38508 & 0.88045 \\
\hline & 9 & 0.66060 & 0.39199 & 0.92922 & 0.66067 & 0.39201 & 0.92933 \\
\hline & 10 & 0.68623 & 0.39829 & 0.97417 & 0.68643 & 0.39838 & 0.97449 \\
\hline
\end{tabular}

TABLE 27: Results of the IT2XB validation index for data clustering of image shown in Figure 2(a) using IT2FPCM and IT2FCM algorithms with $m=[1.5,2.5]$ and $\eta=[1.5,2.5]$ as parameters.

\begin{tabular}{|c|c|c|c|c|c|c|c|}
\hline \multicolumn{8}{|c|}{ Index validation IT2XB } \\
\hline \multirow{2}{*}{ Image } & \multirow{2}{*}{ Clusters } & \multicolumn{3}{|c|}{ IT2FPCM } & \multicolumn{3}{|c|}{ IT2FCM } \\
\hline & & Defuzz & Lower & Upper & Defuzz & Lower & Upper \\
\hline \multirow{9}{*}{ Figure 2(a) } & 2 & 0.11253 & 0.07812 & 0.14694 & 0.11253 & 0.07812 & 0.14694 \\
\hline & 3 & 0.11105 & 0.06516 & 0.15695 & 0.11105 & 0.06516 & 0.15694 \\
\hline & 4 & 0.11980 & 0.06082 & 0.17877 & 0.11979 & 0.06082 & 0.17877 \\
\hline & 5 & 0.11078 & 0.05214 & 0.16943 & 0.11078 & 0.05214 & 0.16943 \\
\hline & 6 & 0.11347 & 0.04983 & 0.17711 & 0.11347 & 0.04983 & 0.17711 \\
\hline & 7 & 0.11478 & 0.04732 & 0.18224 & 0.11478 & 0.04732 & 0.18223 \\
\hline & 8 & 0.12419 & 0.04862 & 0.19975 & 0.12418 & 0.04862 & 0.19974 \\
\hline & 9 & 0.12270 & 0.04573 & 0.19967 & 0.12257 & 0.04567 & 0.19946 \\
\hline & 10 & 0.12837 & 0.04641 & 0.21032 & 0.12848 & 0.04643 & 0.21053 \\
\hline
\end{tabular}

accepts the null hypothesis posed in (15), demonstrating that there is no significant difference between the IT2FCM and IT2FPCM algorithms using the $z$-test for the defuzzification for the IT2XB index.

Also we test both algorithms using images and perform 30 experiments validating the results with each one of the validation indices, in order to observe the behavior of the algorithm performing image segmentation. In this case for the image segmentation with the IT2FPCM and IT2FCM algorithms we perform the steps shown in Figure 1. Using these steps we are capable of making a segmentation of the image using the mentioned above algorithms. The images used for these experiments are shown in Figure 2.

In Tables 25, 26, and 27 we can observe the averages of the IT2MPE-DFPM, IT2PE, and IT2XB indices of validation, respectively, for 2 to 10 clusters, computed with the results 


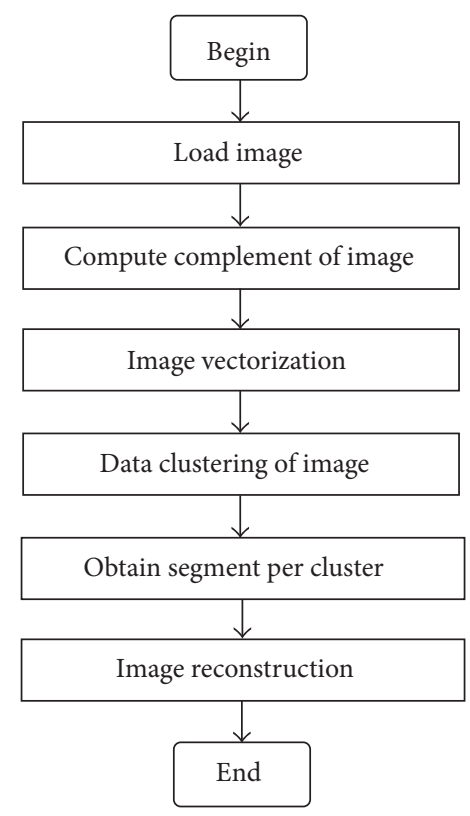

FIGURE 1: Block diagram for segmentation images using clustering algorithms.

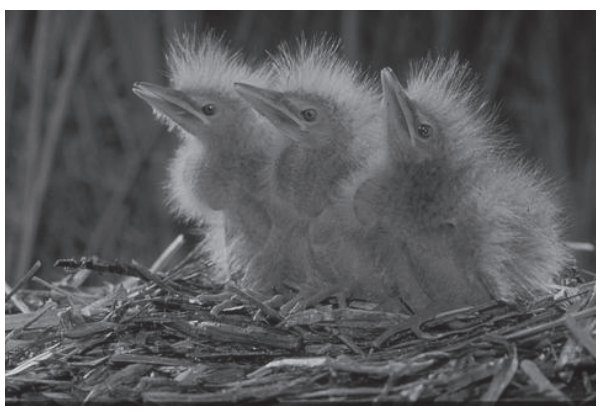

(a)

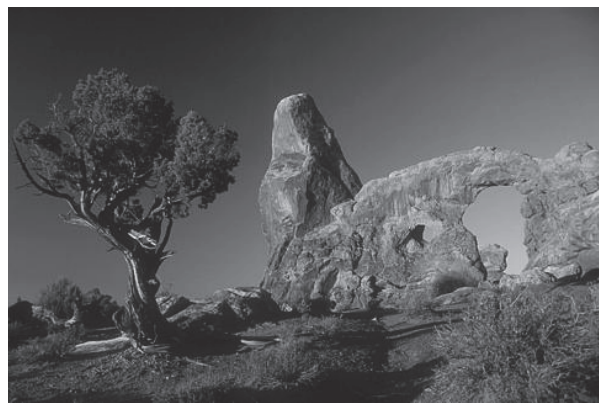

(d)

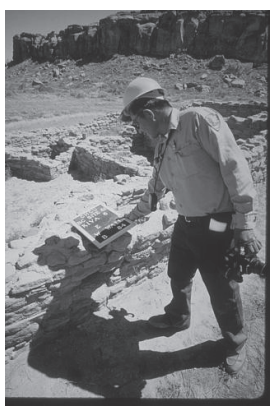

(b)

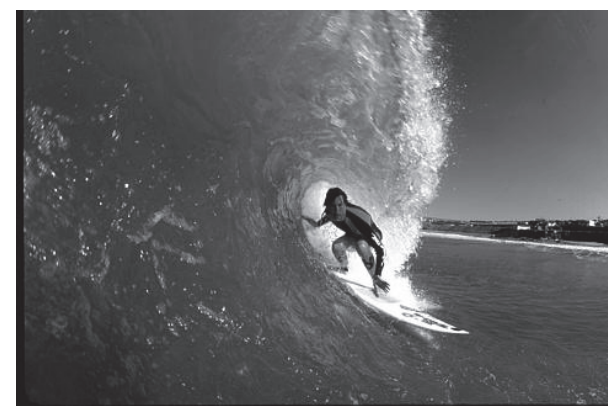

(c)

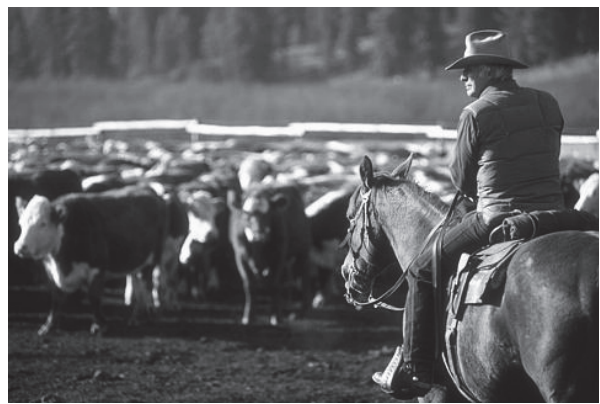

(e)

FIGURE 2: Images for segmentations using the IT2FPCM and IT2FCM algorithms.

(defuzzification, lower and upper bounds of the interval) obtained by the IT2FPCM and IT2FCM algorithms of Figure 2(a). These tables show the number of clusters for the defuzzification, upper and lower, that each validation index found as the best one. Table 28 shows the hypothesis test for the 30 experiments performed in order to know if there exists significant difference between the algorithms using as null and alternative hypothesis the assumptions made in (15).
According to the assumptions made in (15), in Table 28, we can observe for IT2PE and IT2MPE-DMFP indices with the $z$-value of -2342856728 and -232549065.42 , respectively, the hypothesis test that these indices are lower than the $z$-critical value that is equal to -1.645 with a significance level $\alpha$ of 0.05 , whose $z$-value confirms the acceptance of the alternative hypothesis posed in (15), demonstrating that the IT2FPCM algorithm is better than IT2FCM algorithm 
TABLE 28: Hypothesis test for IT2PE, IT2XB, and IT2MPE-DMFP indices of validation for Figure 2(a) clustering.

\begin{tabular}{lcccccccc}
\hline Dataset & Validation index & Algorithm & $N$ & $\mu$ & $\sigma^{2}$ & $z$-value & $z$-critical value $P$ value \\
\hline \multirow{6}{*}{ Figure 2(a) } & IT2PE & IT2FCM & \multirow{2}{*}{30} & 0.27753175 & $4.39 E-28$ & -2342856728 & 1.645 \\
& & IT2FPCM & & 0.27751942 & $3.64 E-28$ & & 0 \\
& \multirow{2}{*}{ IT2XB } & IT2FCM & \multirow{2}{*}{30} & 0.11078111 & $1.76 E-16$ & 361.5239129 & 1.645 \\
& & IT2FPCM & & 0.11078219 & $9.16 E-17$ & & 1 \\
& \multirow{2}{*}{ IT2MPE-DMFP } & IT2FCM & \multirow{2}{*}{30} & 0.31331411 & $4.92 E-26$ & -232549065.42 & 1.645 \\
& IT2FPCM & & 0.31330165 & $3.40 E-26$ & & 0 \\
\hline
\end{tabular}

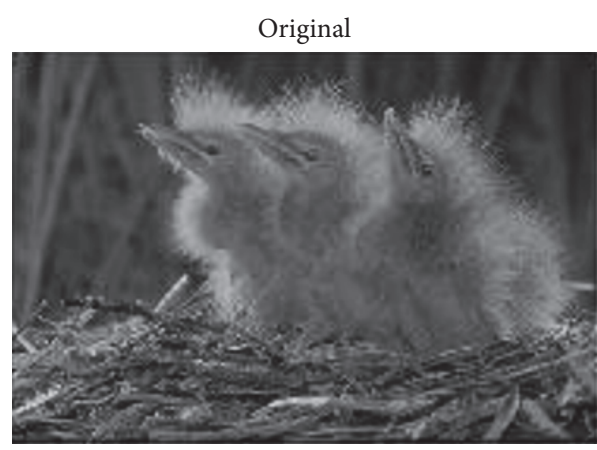

Image lower bound

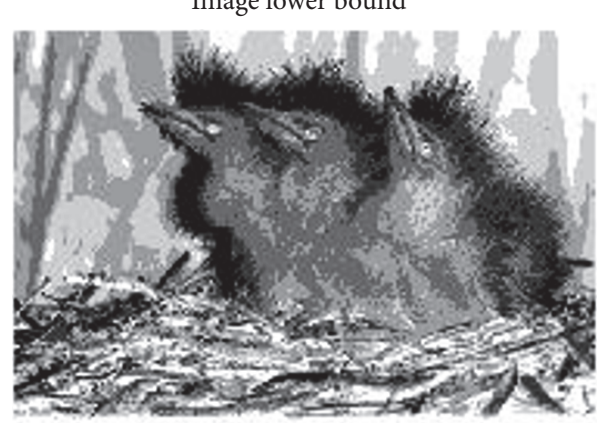

FIGURE 3: Resulting image clustering performed to Figure 2(a) by the IT2FPCM algorithm.

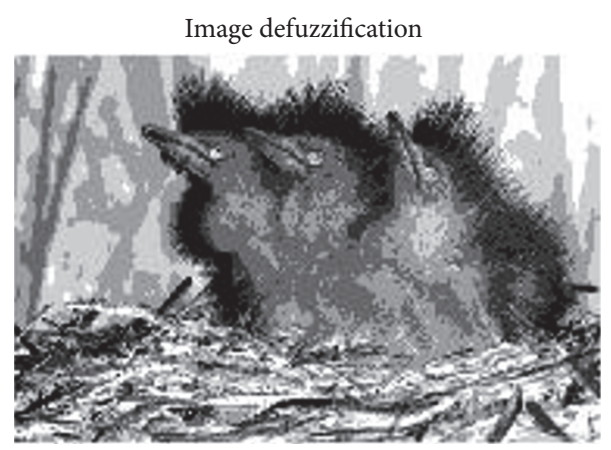

Image upper bound

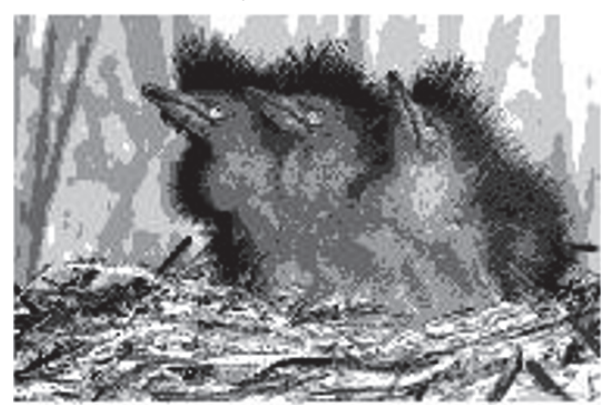

to $z$-test of the defuzzification according to IT2MPE-DMFP and IT2PE indices of validation index for the cluster found by the algorithms in the image shown in Figure 2(a). Also we observe that $z$-value for the IT2XB is 361.5239129 which is greater than the $z$-critical value that is equal to -1.645 and with this information the null hypothesis is accepted demonstrating that the IT2FPCM algorithm is not better than IT2FCM. Figure 3 shows the resulting image clustering performed by the IT2FPCM algorithm for 6 clusters for Figure 2(a) and this is because the gray levels containing the image.

In Tables 29, 30, and 31 we can observe the averages of the IT2MPE-DFPM, IT2PE, and IT2XB indices of validation respectively, for 2 to 10 clusters, computed with the results (defuzzification, lower and upper bounds of the interval) obtained by the IT2FPCM and IT2FCM algorithms for Figure 2(b). These tables show the number of clusters for the defuzzification, upper and lower, that each validation index found like better.

Table 32 shows the hypothesis test for the 30 experiments performed for each index validation mentioned in Section 4 , in order to know if there exists significant difference between the algorithms by using as the null and alternative hypothesis the assumptions made in (15).

In Table 32 we can observe that $z$-values for the hypothesis test of the IT2PE and IT2MPE-DMPF are -10078102681 and -2621392303.80 , respectively, which are lower than the $z$ critical value that is equal to -1.645 with a significance level $\alpha$ of 0.05 , whose $z$-value confirms the acceptance of the alternative hypothesis posed in (15), demonstrating that the IT2FPCM algorithm is better than the IT2FCM algorithm for the $z$-test of the defuzzification according to IT2PE and IT2MPE-DMFP indices of validation indices for the clusters found by the algorithms in the image shown in Figure 2(b). Also we can observe that the $z$-value for the IT2XB validation index is 139756984.7 , which is greater than the $z$-critical value that is equal to -1.645 and with this information the null hypothesis is accepted demonstrating that the IT2FPCM algorithm is not better than IT2FCM according to IT2XB validation index. Figure 4 shows the resulting image clustering performed by the IT2FPCM algorithm for 5 clusters to Figure 2(b) and this is because of the gray levels containing the image. 
TABLE 29: Results of the IT2MPE-DMFP validation index to data clustering of image shown in Figure 2(b) using IT2FPCM and IT2FCM algorithm with $m=[1.5,2.5]$ and $\eta=[1.5,2.5]$ as parameters.

\begin{tabular}{|c|c|c|c|c|c|c|c|}
\hline \multicolumn{8}{|c|}{ Index of validation IT2MPEDFP } \\
\hline \multirow{2}{*}{ Image } & \multirow{2}{*}{ Clusters } & \multicolumn{3}{|c|}{ IT2FPCM } & \multicolumn{3}{|c|}{ IT2FCM } \\
\hline & & Defuzz & Lower & Upper & Defuzz & Lower & Upper \\
\hline \multirow{9}{*}{ Figure 2(b) } & 2 & 0.20780 & 0.22583 & 0.18978 & 0.20781 & 0.22584 & 0.18979 \\
\hline & 3 & 0.35850 & 0.37345 & 0.34355 & 0.35852 & 0.37346 & 0.34358 \\
\hline & 4 & 0.54660 & 0.54745 & 0.54574 & 0.54662 & 0.54748 & 0.54577 \\
\hline & 5 & 0.84354 & 0.81276 & 0.87432 & 0.84357 & 0.81278 & 0.87436 \\
\hline & 6 & 0.76870 & 0.72207 & 0.81534 & 0.76874 & 0.72208 & 0.81540 \\
\hline & 7 & 0.91927 & 0.84436 & 0.99419 & 0.91977 & 0.84473 & 0.99480 \\
\hline & 8 & 1.21142 & 1.07707 & 1.34578 & 1.20963 & 1.07551 & 1.34375 \\
\hline & 9 & 1.16203 & 1.02427 & 1.29979 & 1.16137 & 1.02366 & 1.29909 \\
\hline & 10 & 1.51477 & 1.30270 & 1.72684 & 1.51163 & 1.29979 & 1.72348 \\
\hline
\end{tabular}

TABLE 30: Results of the IT2PE validation index to the clustering of image shown in Figure 2(b) using IT2FPCM and IT2FCM algorithm with $m=[1.5,2.5]$ and $\eta=[1.5,2.5]$ as parameters.

\begin{tabular}{|c|c|c|c|c|c|c|c|}
\hline \multicolumn{8}{|c|}{ Index of validation IT2PE } \\
\hline \multirow{2}{*}{ Image } & \multirow{2}{*}{ Clusters } & \multicolumn{3}{|c|}{ IT2FPCM } & \multicolumn{3}{|c|}{ IT2FCM } \\
\hline & & Defuzz & Lower & Upper & Defuzz & Lower & Upper \\
\hline \multirow{9}{*}{ Figure 2(b) } & 2 & 0.22538 & 0.18895 & 0.26181 & 0.22539 & 0.18895 & 0.26183 \\
\hline & 3 & 0.32425 & 0.24288 & 0.40561 & 0.32427 & 0.24290 & 0.40564 \\
\hline & 4 & 0.39445 & 0.27438 & 0.51452 & 0.39447 & 0.27440 & 0.51455 \\
\hline & 5 & 0.44970 & 0.29589 & 0.60351 & 0.44973 & 0.29591 & 0.60355 \\
\hline & 6 & 0.51026 & 0.32817 & 0.69236 & 0.51030 & 0.32819 & 0.69240 \\
\hline & 7 & 0.55406 & 0.34735 & 0.76078 & 0.55410 & 0.34737 & 0.76083 \\
\hline & 8 & 0.58858 & 0.36076 & 0.81639 & 0.58866 & 0.36084 & 0.81649 \\
\hline & 9 & 0.61605 & 0.36984 & 0.86225 & 0.61608 & 0.36987 & 0.86229 \\
\hline & 10 & 0.63779 & 0.37485 & 0.90074 & 0.63801 & 0.37496 & 0.90107 \\
\hline
\end{tabular}

TABLE 31: Results of the IT2XB validation index to the clustering of image shown in Figure 2(b) using IT2FPCM and IT2FCM algorithm with $m=[1.5,2.5]$ and $\eta=[1.5,2.5]$ as parameters.

\begin{tabular}{|c|c|c|c|c|c|c|c|}
\hline \multicolumn{8}{|c|}{ Index of validation IT2XB } \\
\hline \multirow{2}{*}{ Image } & \multirow{2}{*}{ Clusters } & \multicolumn{3}{|c|}{ IT2FPCM } & \multicolumn{3}{|c|}{ IT2FCM } \\
\hline & & Defuzz & Lower & Upper & Defuzz & Lower & Upper \\
\hline \multirow{9}{*}{ Figure 2(b) } & 2 & 0.05906 & 0.04269 & 0.07544 & 0.05906 & 0.04269 & 0.07544 \\
\hline & 3 & 0.06108 & 0.03734 & 0.08483 & 0.06108 & 0.03734 & 0.08483 \\
\hline & 4 & 0.06307 & 0.03463 & 0.09150 & 0.06307 & 0.03463 & 0.09150 \\
\hline & 5 & 0.06633 & 0.03419 & 0.09846 & 0.06633 & 0.03419 & 0.09846 \\
\hline & 6 & 0.10278 & 0.04910 & 0.15646 & 0.10278 & 0.04910 & 0.15646 \\
\hline & 7 & 0.11868 & 0.05438 & 0.18298 & 0.11862 & 0.05434 & 0.18289 \\
\hline & 8 & 0.11918 & 0.05262 & 0.18574 & 0.11938 & 0.05273 & 0.18604 \\
\hline & 9 & 0.14904 & 0.06355 & 0.23454 & 0.14913 & 0.06358 & 0.23468 \\
\hline & 10 & 0.13597 & 0.05632 & 0.21562 & 0.13620 & 0.05641 & 0.21599 \\
\hline
\end{tabular}

In Tables 33, 34, and 35 we can observe the averages of the IT2MPE-DFPM, IT2PE, and IT2XB indices of validation, respectively, for 2 to 10 clusters, computed with the results (defuzzification, lower and upper bounds of the interval) obtained by the IT2FPCM and IT2FCM algorithms to Figure 2(c). These tables show the number of clusters for the defuzzification, the upper and lower values that each validation index found like better.

In Table 36, we can observe that $z$-values to the hypothesis test of the IT2PE and IT2MPE-DMPF are -7686717373 and -1117084835.71 , respectively, which are less than the $z$-critical value that is equal to -1.645 with a significant level $(\alpha)$ of 
TABLE 32: Hypothesis test for IT2PE, IT2XB, and IT2MPE-DMFP indices of validation for Figure 2(b) clustering.

\begin{tabular}{lcccccccc}
\hline Dataset & Validation index & Algorithm & $N$ & $\mu$ & $\sigma^{2}$ & $z$-value & $z$-critical value $P$ value \\
\hline \multirow{6}{*}{ Figure 2(b) } & IT2PE & IT2FCM & \multirow{2}{*}{30} & 0.22539012 & $4.13 E-29$ & -10078102681 & 1.645 \\
& & IT2FPCM & & 0.2253774 & $6.51 E-30$ & & 0 \\
& \multirow{2}{*}{ IT2XB } & IT2FCM & \multirow{2}{*}{30} & 0.05906138 & $7.70 E-30$ & 139756984.7 & 1.645 \\
& IT2FPCM & & 0.05906146 & $2.49 E-30$ & & 1 \\
& IT2MP-DMFP & IT2FCM & \multirow{2}{*}{30} & 0.20781375 & $3.03 E-28$ & -2621392303.80 & 1.645 \\
& & IT2FPCM & & 0.20780095 & $4.13 E-28$ & & 0 \\
\hline
\end{tabular}
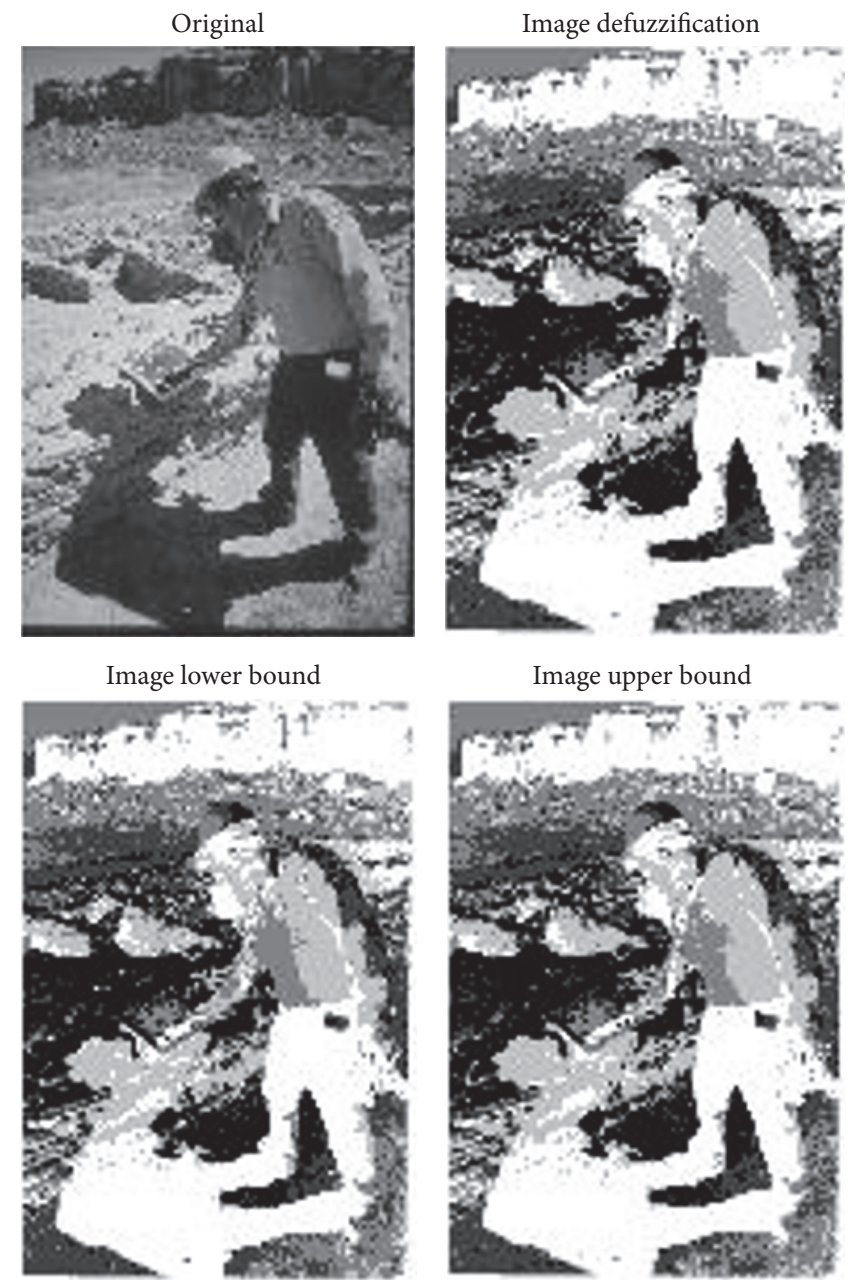

FIGURE 4: Resulting image clustering performed by the IT2FPCM algorithm for 5 clusters to Figure 2(b) because of the gray levels containing the image.

0.05 , whose $z$-value confirms the acceptance of the alternative hypothesis posed in (15), demonstrating that IT2FPCM algorithm is better than IT2FCM algorithm to $z$-test of the defuzzification according to IT2PE and IT2MPE-DMFP indices of validation index for the cluster found by the algorithms in image shown in Figure 2(c). Also we can observe that $z$-value to the IT2XB validation index is 63042445.95, which is greater than the $z$-critical value that is equal to -1.645 ; with this information the null hypothesis is accepted demonstrating that the IT2FPCM algorithm is not better than the IT2FCM according to the IT2XB validation index.
Figure 5 shows the resulting image clustering performed by the IT2FPCM algorithm for 5 clusters to Figure 2(c) because of the gray levels containing the image.

In Tables 37, 38, and 39 we can observe the averages of the IT2MPE-DFPM, IT2PE, and IT2XB indices of validation, respectively, for 2 to 10 clusters, computed with the results (defuzzification, lower and upper bounds of the interval) obtained by the IT2FPCM and IT2FCM algorithms to Figure 2(d). These tables show the number of clusters for the defuzzification, the upper and lower values that each validation index found like better. 


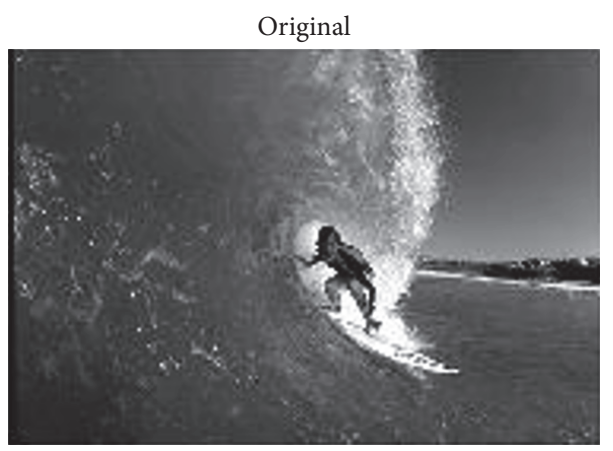

Image lower bound

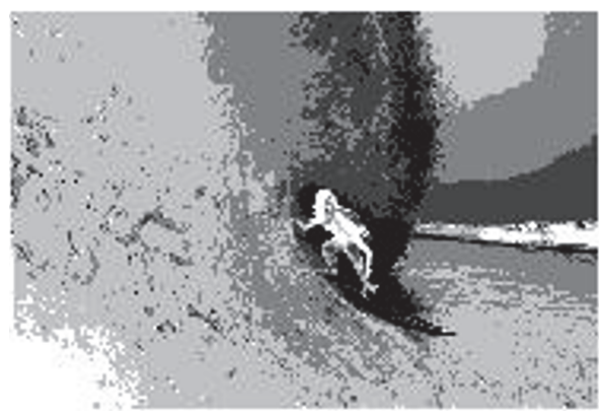

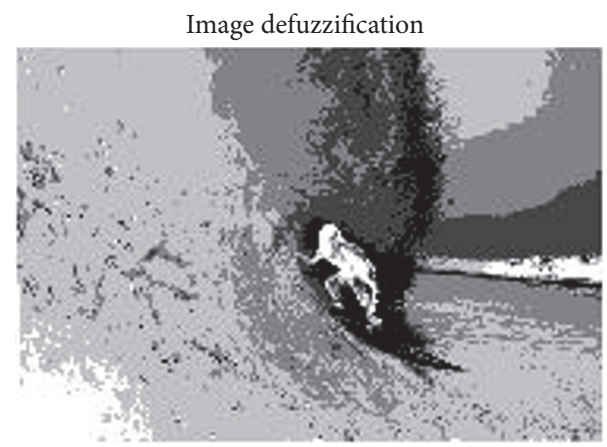

Image upper bound

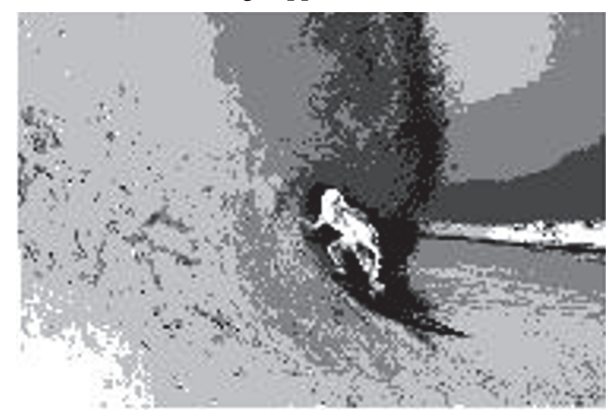

FIGURE 5: Resulting image clustering performed by the IT2FPCM algorithm for 5 clusters to Figure 2(c) because of the gray levels containing the image.

TABLE 33: Results of the IT2MPE-DMFP validation index to data clustering of image shown in Figure 2(c) using IT2FPCM and IT2FCM algorithm with $m=[1.5,2.5]$ and $\eta=[1.5,2.5]$ as parameters.

\begin{tabular}{|c|c|c|c|c|c|c|c|}
\hline \multicolumn{8}{|c|}{ Index of validation IT2MPEDFP } \\
\hline \multirow{2}{*}{ Image } & \multirow{2}{*}{ Clusters } & \multicolumn{3}{|c|}{ IT2FPCM } & \multicolumn{3}{|c|}{ IT2FCM } \\
\hline & & Defuzz & Lower & Upper & Defuzz & Lower & Upper \\
\hline \multirow{9}{*}{ Figure 2(c) } & 2 & 0.65158 & 0.66791 & 0.63526 & 0.65159 & 0.66791 & 0.63528 \\
\hline & 3 & 1.19860 & 1.14601 & 1.25119 & 1.19862 & 1.14601 & 1.25123 \\
\hline & 4 & 1.37171 & 1.21107 & 1.53235 & 1.37174 & 1.21109 & 1.53239 \\
\hline & 5 & 2.11610 & 1.87005 & 2.36214 & 2.11613 & 1.87006 & 2.36219 \\
\hline & 6 & 2.55874 & 2.21054 & 2.90695 & 2.55876 & 2.21054 & 2.90699 \\
\hline & 7 & 2.87029 & 2.40246 & 3.33812 & 2.87033 & 2.40247 & 3.33820 \\
\hline & 8 & 3.26360 & 2.68131 & 3.84588 & 3.26782 & 2.68456 & 3.85108 \\
\hline & 9 & 3.67714 & 2.97374 & 4.38054 & 3.62469 & 2.92623 & 4.32314 \\
\hline & 10 & 4.14291 & 3.29282 & 4.99301 & 4.13626 & 3.28607 & 4.98644 \\
\hline
\end{tabular}

In Table 40, we can observe that $z$-values to the hypothesis test of the IT2PE and IT2MPE-DMPF are $-4.95875 E+13$ and -3341752881.89 , respectively, which are less than the $z$-critical value that is equal to -1.645 with a significant level $(\alpha)$ of 0.05 , whose $z$-value confirms the acceptance of the alternative hypothesis posed in (15), demonstrating that IT2FPCM algorithm is better than IT2FCM algorithm to $z$ test of the defuzzification according to IT2PE and IT2MPEDMFP indices of validation index for the cluster found by the algorithms in image shown in Figure 2(d). Also we can observe that $z$-value to the IT2XB validation index is 16637443.08 , which is greater than the $z$-critical value that is equal to -1.645 ; with this information the null hypothesis is accepted demonstrating that the IT2FPCM algorithm is not better than the IT2FCM according to the IT2XB validation index. Figure 6 shows the resulting image clustering performed by the IT2FPCM algorithm for 7 clusters to Figure 2(d) because of the gray levels containing the image.

In Tables 41, 42, and 43 we can observe the averages of the IT2MPE-DFPM, IT2PE, and IT2XB indices of validation, respectively, for 2 to 10 clusters, computed with the results (defuzzification, lower and upper bounds of the interval) obtained by the IT2FPCM and IT2FCM algorithms to Figure 2(e). These tables show the number of clusters for the defuzzification, the upper and lower values that each validation index found like better.

In Table 44, we can observe that $z$-values to the hypothesis test of the IT2PE and IT2MPE-DMPF are -23529815362 
TABLE 34: Results of the IT2PE validation index to the clustering of image shown in Figure 2(c) using IT2FPCM and IT2FCM algorithm with $m=[1.5,2.5]$ and $\eta=[1.5,2.5]$ as parameters.

\begin{tabular}{|c|c|c|c|c|c|c|c|}
\hline \multirow{3}{*}{ Image } & \multirow{3}{*}{ Clusters } & \multicolumn{3}{|c|}{ Index of validation IT2PE } & \multirow{2}{*}{\multicolumn{3}{|c|}{ IT2FCM }} \\
\hline & & & IT2FPCM & & & & \\
\hline & & Defuzz & Lower & Upper & Defuzz & Lower & Upper \\
\hline \multirow{9}{*}{ Figure 2(c) } & 2 & 0.19464 & 0.16113 & 0.22815 & 0.19465 & 0.16114 & 0.22817 \\
\hline & 3 & 0.33148 & 0.25917 & 0.40380 & 0.33150 & 0.25917 & 0.40383 \\
\hline & 4 & 0.43944 & 0.32977 & 0.54911 & 0.43947 & 0.32978 & 0.54915 \\
\hline & 5 & 0.47479 & 0.32064 & 0.62893 & 0.47481 & 0.32066 & 0.62897 \\
\hline & 6 & 0.52781 & 0.34398 & 0.71165 & 0.52784 & 0.34399 & 0.71169 \\
\hline & 7 & 0.55923 & 0.35528 & 0.76319 & 0.55927 & 0.35530 & 0.76323 \\
\hline & 8 & 0.58906 & 0.36326 & 0.81485 & 0.58911 & 0.36333 & 0.81489 \\
\hline & 9 & 0.62291 & 0.37466 & 0.87116 & 0.62580 & 0.37766 & 0.87394 \\
\hline & 10 & 0.64876 & 0.38289 & 0.91463 & 0.64964 & 0.38373 & 0.91555 \\
\hline
\end{tabular}

TABLE 35: Results of the IT2XB validation index to the clustering of image shown in Figure 2(c) using IT2FPCM and IT2FCM algorithm with $m=[1.5,2.5]$ and $\eta=[1.5,2.5]$ as parameters.

\begin{tabular}{|c|c|c|c|c|c|c|c|}
\hline \multicolumn{8}{|c|}{ Index of validation IT2XB } \\
\hline \multirow{2}{*}{ Image } & \multirow{2}{*}{ Clusters } & \multicolumn{3}{|c|}{ IT2FPCM } & \multicolumn{3}{|c|}{ IT2FCM } \\
\hline & & Defuzz & Lower & Upper & Defuzz & Lower & Upper \\
\hline \multirow{9}{*}{ Figure 2(c) } & 2 & 0.07255 & 0.04993 & 0.09517 & 0.07255 & 0.04993 & 0.09517 \\
\hline & 3 & 0.12235 & 0.07282 & 0.17187 & 0.12235 & 0.07282 & 0.17187 \\
\hline & 4 & 0.20659 & 0.11269 & 0.30048 & 0.20658 & 0.11269 & 0.30048 \\
\hline & 5 & 0.14144 & 0.07037 & 0.21252 & 0.14144 & 0.07037 & 0.21252 \\
\hline & 6 & 0.14983 & 0.07128 & 0.22839 & 0.14983 & 0.07128 & 0.22838 \\
\hline & 7 & 0.18642 & 0.08317 & 0.28966 & 0.18641 & 0.08317 & 0.28965 \\
\hline & 8 & 0.18905 & 0.08034 & 0.29777 & 0.18869 & 0.08021 & 0.29718 \\
\hline & 9 & 0.18358 & 0.07500 & 0.29216 & 0.18937 & 0.07724 & 0.30150 \\
\hline & 10 & 0.18118 & 0.07167 & 0.29068 & 0.18252 & 0.07222 & 0.29283 \\
\hline
\end{tabular}

TABLE 36: Statistical test for the IT2PE, IT2XB, and IT2MPE-DMFP indices of validation for Figure 2(c) clustering.

\begin{tabular}{lcccccccc}
\hline Dataset & Validation index & Algorithm & $N$ & $\mu$ & $\sigma^{2}$ & $z$-value & $z$-critical value $P$ value \\
\hline \multirow{6}{*}{ Figure 2(c) } & IT2PE & IT2FCM & \multirow{2}{*}{30} & 0.19465271 & $6.95 E-29$ & -7686717373 & 1.645 \\
& & IT2FPCM & & 0.19464102 & $1.23 E-32$ & & 0 \\
& IT2XB & IT2FCM & \multirow{2}{*}{30} & 0.07255222 & $2.29 E-28$ & 63042445.95 & 1.645 \\
& & IT2FPCM & & 0.0725524 & $1.52 E-32$ & & 1 \\
& IT2MPE-DMFP & IT2FCM & \multirow{2}{*}{30} & 0.65159414 & $3.67 E-27$ & -1117084835.71 & 1.645 \\
& & IT2FPCM & & 0.65158179 & $2.23 E-31$ & & 0 \\
\hline
\end{tabular}

TABLE 37: Results of the IT2MPE-DMFP validation index to data clustering of image shown in Figure 2(d) using IT2FPCM and IT2FCM algorithm with $m=[1.5,2.5]$ and $\eta=[1.5,2.5]$ as parameters.

\begin{tabular}{|c|c|c|c|c|c|c|c|}
\hline \multicolumn{8}{|c|}{ Index of validation IT2MPEDFP } \\
\hline \multirow{2}{*}{ Image } & \multirow{2}{*}{ Clusters } & \multicolumn{3}{|c|}{ IT2FPCM } & \multicolumn{3}{|c|}{ IT2FCM } \\
\hline & & Defuzz & Lower & Upper & Defuzz & Lower & Upper \\
\hline \multirow{9}{*}{ Figure 2(d) } & 2 & 0.15969 & 0.17663 & 0.14274 & 0.15970 & 0.17663 & 0.14276 \\
\hline & 3 & 0.52574 & 0.50323 & 0.54825 & 0.52576 & 0.50324 & 0.54828 \\
\hline & 4 & 0.67185 & 0.63511 & 0.70860 & 0.67188 & 0.63511 & 0.70865 \\
\hline & 5 & 0.92726 & 0.82782 & 1.02669 & 0.92729 & 0.82782 & 1.02675 \\
\hline & 6 & 0.98403 & 0.84965 & 1.11841 & 0.98406 & 0.84964 & 1.11849 \\
\hline & 7 & 1.30797 & 1.11585 & 1.50009 & 1.30804 & 1.11588 & 1.50020 \\
\hline & 8 & 1.39216 & 1.12362 & 1.66071 & 1.39200 & 1.12350 & 1.66050 \\
\hline & 9 & 1.75595 & 1.40758 & 2.10433 & 1.76502 & 1.41523 & 2.11480 \\
\hline & 10 & 1.98371 & 1.56297 & 2.40445 & 1.98233 & 1.56168 & 2.40299 \\
\hline
\end{tabular}


TABLE 38: Results of the IT2PE validation index to the clustering of image shown in Figure 2(d) using IT2FPCM and IT2FCM algorithm with $m=[1.5,2.5]$ and $\eta=[1.5,2.5]$ as parameters.

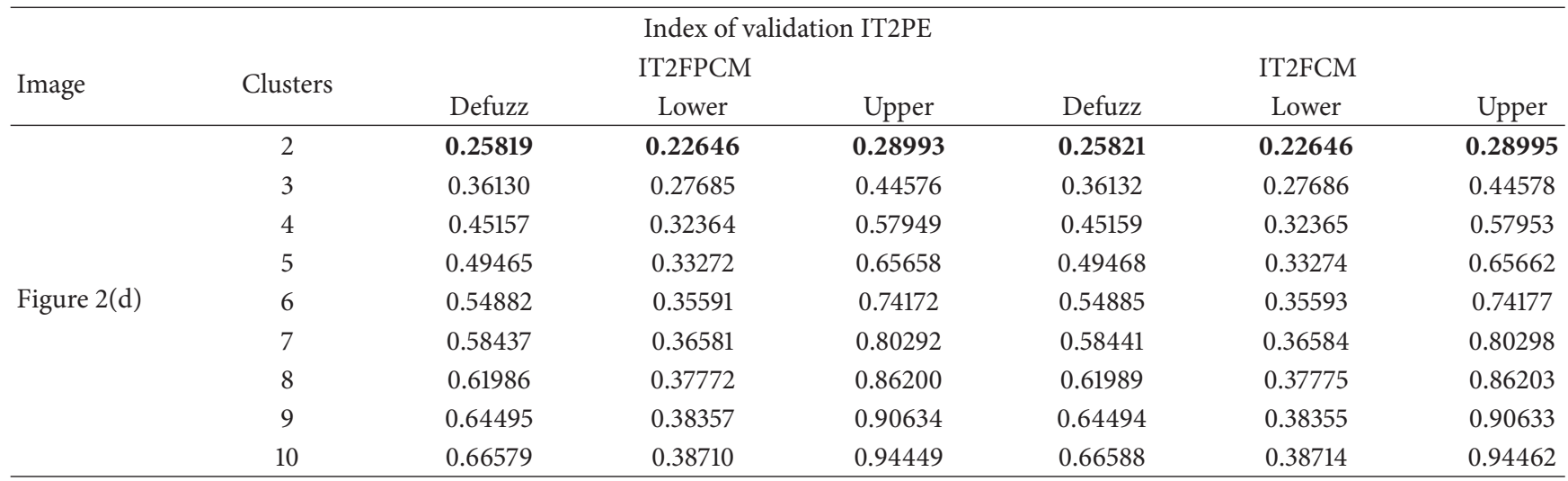

TABLE 39: Results of the IT2XB validation index to the clustering of image shown in Figure 2(d) using IT2FPCM and IT2FCM algorithm with $m=[1.5,2.5]$ and $\eta=[1.5,2.5]$ as parameters.

\begin{tabular}{|c|c|c|c|c|c|c|c|}
\hline \multicolumn{8}{|c|}{ Index validation IT2XB } \\
\hline \multirow{2}{*}{ Image } & \multirow{2}{*}{ Clusters } & \multicolumn{3}{|c|}{ IT2FPCM } & \multicolumn{3}{|c|}{ IT2FCM } \\
\hline & & Defuzz & Lower & Upper & Defuzz & Lower & Upper \\
\hline \multirow{9}{*}{ Figure 2(d) } & 2 & 0.09227 & 0.06428 & 0.12026 & 0.09227 & 0.06428 & 0.12026 \\
\hline & 3 & 0.07539 & 0.04451 & 0.10626 & 0.07539 & 0.04451 & 0.10626 \\
\hline & 4 & 0.09492 & 0.05034 & 0.13951 & 0.09492 & 0.05034 & 0.13950 \\
\hline & 5 & 0.08523 & 0.04182 & 0.12864 & 0.08523 & 0.04182 & 0.12864 \\
\hline & 6 & 0.10438 & 0.04713 & 0.16164 & 0.10438 & 0.04713 & 0.16164 \\
\hline & 7 & 0.09127 & 0.04010 & 0.14244 & 0.09126 & 0.04010 & 0.14243 \\
\hline & 8 & 0.10893 & 0.04570 & 0.17217 & 0.10897 & 0.04571 & 0.17223 \\
\hline & 9 & 0.10584 & 0.04343 & 0.16825 & 0.10532 & 0.04326 & 0.16738 \\
\hline & 10 & 0.10343 & 0.04109 & 0.16577 & 0.10347 & 0.04111 & 0.16584 \\
\hline
\end{tabular}

TABLE 40: Statistical test for the IT2PE, IT2XB, and IT2MPE-DMFP indices of validation for Figure 2(d) clustering.

\begin{tabular}{lcccccccc}
\hline Dataset & Validation index & Algorithm & $N$ & $\mu$ & $\sigma^{2}$ & $z$-value & $z$-critical value & $P$ value \\
\hline \multirow{6}{*}{ Figure 2(d) } & \multirow{2}{*}{ IT2PE } & IT2FCM & \multirow{2}{*}{30} & 0.25820712 & $8.76 E-30$ & $-4.95875 E+13$ & 1.645 \\
& & IT2FPCM & & 0.2581946 & $3.35 E-30$ & & \\
& \multirow{2}{*}{ IT2XB } & IT2FCM & \multirow{2}{*}{30} & 0.07538511 & $6.34 E-27$ & 16637443.08 & 1.645 \\
& & IT2FPCM & & 0.07538535 & $1.30 E-28$ & & 1 \\
& \multirow{2}{*}{ IT2MPE-DMFP } & IT2FCM & \multirow{2}{*}{30} & 0.15969978 & $3.39 E-30$ & -3341752881.89 & 1.645 \\
& & IT2FPCM & & 0.15968716 & $4.25 E-28$ & & 0 \\
\hline
\end{tabular}

TABLE 41: Results of the IT2MPE-DMFP validation index to data clustering of image shown in Figure 2(e) using IT2FPCM and IT2FCM algorithm with $m=[1.5,2.5]$ and $\eta=[1.5,2.5]$ as parameters.

\begin{tabular}{|c|c|c|c|c|c|c|c|}
\hline \multicolumn{8}{|c|}{ Index of validation IT2MPEDFP } \\
\hline \multirow{2}{*}{ Image } & \multirow{2}{*}{ Clusters } & \multicolumn{3}{|c|}{ IT2FPCM } & \multicolumn{3}{|c|}{ IT2FCM } \\
\hline & & Defuzz & Lower & Upper & Defuzz & Lower & Upper \\
\hline \multirow{9}{*}{ Figure 2(e) } & 2 & 0.39275 & 0.41011 & 0.37538 & 0.39276 & 0.41011 & 0.37541 \\
\hline & 3 & 0.82005 & 0.79494 & 0.84516 & 0.82007 & 0.79494 & 0.84519 \\
\hline & 4 & 0.93366 & 0.85748 & 1.00984 & 0.93368 & 0.85749 & 1.00988 \\
\hline & 5 & 1.46587 & 1.30791 & 1.62383 & 1.46590 & 1.30792 & 1.62388 \\
\hline & 6 & 1.48807 & 1.24435 & 1.73180 & 1.48811 & 1.24438 & 1.73184 \\
\hline & 7 & 1.61212 & 1.33261 & 1.89163 & 1.61217 & 1.33262 & 1.89171 \\
\hline & 8 & 2.20927 & 1.81935 & 2.59920 & 2.20931 & 1.81917 & 2.59946 \\
\hline & 9 & 2.18831 & 1.74485 & 2.63178 & 2.18867 & 1.74513 & 2.63220 \\
\hline & 10 & 2.28302 & 1.78155 & 2.78449 & 2.28147 & 1.78004 & 2.78291 \\
\hline
\end{tabular}




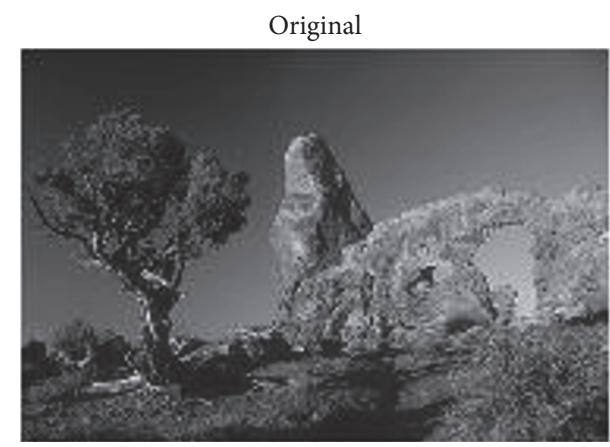

Image lower bound

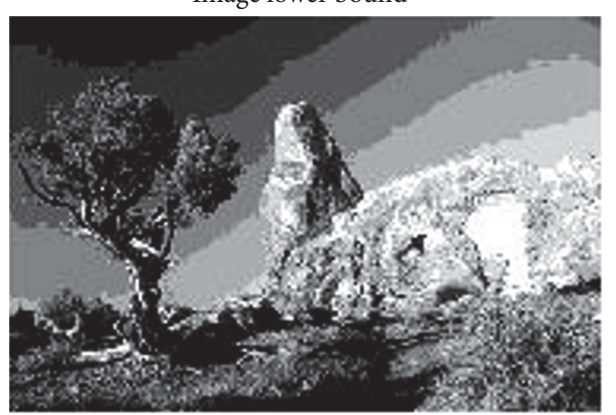

Image defuzzification

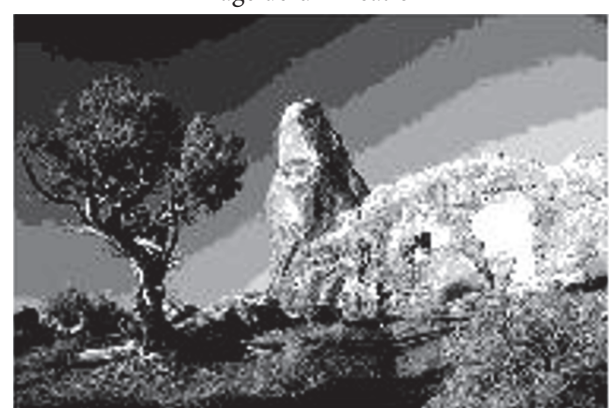

Image upper bound

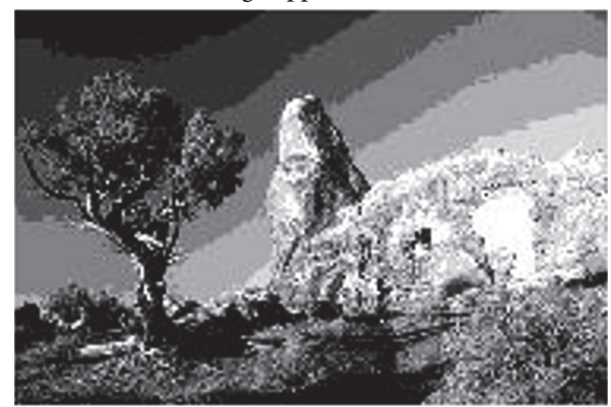

FIGURE 6: The resulting image clustering performed by the IT2FPCM algorithm for 7 clusters to Figure 2(d) because of the gray levels containing the image.

TABLE 42: Results of the IT2PE validation index to the clustering of image shown in Figure 2(e) using IT2FPCM and IT2FCM algorithm with $m=[1.5,2.5]$ and $\eta=[1.5,2.5]$ as parameters.

\begin{tabular}{|c|c|c|c|c|c|c|c|}
\hline \multicolumn{8}{|c|}{ Index of validation IT2PE } \\
\hline \multirow{2}{*}{ Image } & \multirow{2}{*}{ Clusters } & \multicolumn{3}{|c|}{ IT2FPCM } & \multicolumn{3}{|c|}{ IT2FCM } \\
\hline & & Defuzz & Lower & Upper & Defuzz & Lower & Upper \\
\hline \multirow{9}{*}{ Figure 2(e) } & 2 & 0.25722 & 0.22472 & 0.28971 & 0.25723 & 0.22473 & 0.28973 \\
\hline & 3 & 0.34121 & 0.25920 & 0.42321 & 0.34122 & 0.25921 & 0.42324 \\
\hline & 4 & 0.42472 & 0.30360 & 0.54583 & 0.42474 & 0.30362 & 0.54586 \\
\hline & 5 & 0.48178 & 0.32465 & 0.63891 & 0.48181 & 0.32467 & 0.63894 \\
\hline & 6 & 0.53382 & 0.34760 & 0.72005 & 0.53386 & 0.34762 & 0.72010 \\
\hline & 7 & 0.55569 & 0.34695 & 0.76443 & 0.55573 & 0.34698 & 0.76448 \\
\hline & 8 & 0.58706 & 0.35582 & 0.81830 & 0.58706 & 0.35580 & 0.81831 \\
\hline & 9 & 0.62347 & 0.37038 & 0.87656 & 0.62353 & 0.37041 & 0.87665 \\
\hline & 10 & 0.65010 & 0.37895 & 0.92124 & 0.65020 & 0.37899 & 0.92142 \\
\hline
\end{tabular}

and -2455999372.25 , respectively, which are less than the $z$-critical value that is equal to -1.645 with a significant level $(\alpha)$ of 0.05 , whose $z$-value confirms the acceptance of the alternative hypothesis posed in (15), demonstrating that IT2FPCM algorithm is better than IT2FCM algorithm to $z$ test of the defuzzification according to IT2PE and IT2MPEDMFP indices of validation index for the cluster found by the algorithms in image shown in Figure 2(e). Also we can observe that $z$-value to the IT2XB validation index is 305500241.9 , which is greater than the $z$-critical value that is equal to -1.645 ; with this information the null hypothesis is accepted demonstrating that the IT2FPCM algorithm is not better than the IT2FCM according to the IT2XB validation index. Figure 7 shows the resulting image clustering performed by the IT2FPCM algorithm for 7 clusters to Figure 2(e) because of the gray levels containing the image.

\section{Conclusions}

IT2FPCM is an extension of the FPCM algorithm based on Type-2 Fuzzy Logic concepts, in order to enhance its ability of handling uncertainty and making it less susceptible to noise. This algorithm was tested using the Wine, WDBC, Iris Flower, Ionosphere, Abalone, and Cover type benchmark datasets and a set of images shown in Figure 2. In order to observe if the proposal is better than the IT2FCM algorithm we performed 30 experiments with each dataset and images used for a number of clusters from 2 to 10 , in order to make a hypothesis testing with the assumption made in 
TABLE 43: Results of the IT2XB validation index to the clustering of image shown in Figure 2(e) using IT2FPCM and IT2FCM algorithm with $m=[1.5,2.5]$ and $\eta=[1.5,2.5]$ as parameters.

\begin{tabular}{|c|c|c|c|c|c|c|c|}
\hline \multicolumn{8}{|c|}{ Index validation IT2XB } \\
\hline \multirow{2}{*}{ Image } & \multirow{2}{*}{ Clusters } & \multicolumn{3}{|c|}{ IT2FPCM } & \multicolumn{3}{|c|}{ IT2FCM } \\
\hline & & Defuzz & Lower & Upper & Defuzz & Lower & Upper \\
\hline \multirow{9}{*}{ Figure 2(e) } & 2 & 0.09682 & 0.06740 & 0.12625 & 0.09682 & 0.06740 & 0.12625 \\
\hline & 3 & 0.08314 & 0.05171 & 0.11457 & 0.08314 & 0.05171 & 0.11457 \\
\hline & 4 & 0.10315 & 0.05592 & 0.15038 & 0.10315 & 0.05592 & 0.15038 \\
\hline & 5 & 0.08615 & 0.04418 & 0.12812 & 0.08615 & 0.04418 & 0.12811 \\
\hline & 6 & 0.11084 & 0.05313 & 0.16855 & 0.11084 & 0.05313 & 0.16855 \\
\hline & 7 & 0.11129 & 0.05049 & 0.17209 & 0.11129 & 0.05049 & 0.17209 \\
\hline & 8 & 0.09285 & 0.04078 & 0.14492 & 0.09285 & 0.04078 & 0.14491 \\
\hline & 9 & 0.10428 & 0.04261 & 0.16594 & 0.10423 & 0.04261 & 0.16586 \\
\hline & 10 & 0.12035 & 0.04734 & 0.19336 & 0.12045 & 0.04736 & 0.19354 \\
\hline
\end{tabular}

TABLE 44: Statistical test for the IT2PE, IT2XB, and IT2MPE-DMFP indices of validation for Figure 2(e) clustering.

\begin{tabular}{lcccccccc}
\hline Dataset & Validation index & Algorithm & $N$ & $\mu$ & $\sigma^{2}$ & $z$-value & $z$-critical value & $P$ value \\
\hline \multirow{5}{*}{ Figure 2(e) } & \multirow{2}{*}{ IT2PE } & IT2FCM & \multirow{2}{*}{30} & 0.25722842 & $8.46 E-31$ & -23529815362 & 1.645 \\
& & IT2FPCM & & 0.2572161 & $7.37 E-30$ & & 0 \\
& \multirow{2}{*}{ IT2XB } & IT2FCM & \multirow{2}{*}{30} & 0.04078364 & $1.06 E-06$ & 305500241.9 & 1.645 \\
& & IT2FPCM & & 0.04078258 & $1.05 E-06$ & & 1 \\
& \multirow{2}{*}{ IT2MPE-DMFP } & IT2FCM & \multirow{2}{*}{30} & 0.39275846 & $9.74 E-30$ & -2455999372.25 & 1.645 \\
& IT2FPCM & & 0.39274591 & $7.73 E-28$ & & 0 \\
\hline
\end{tabular}
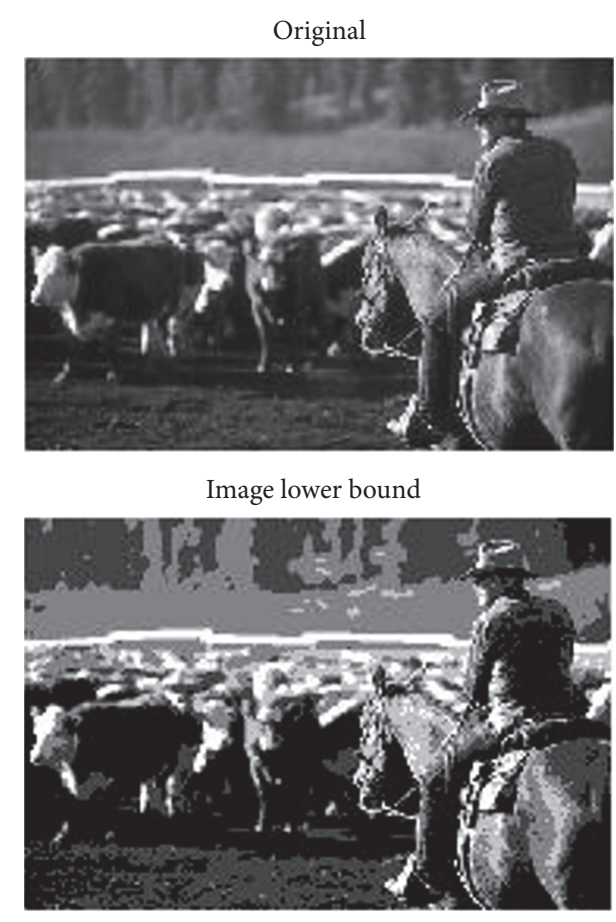

FIGURE 7: Resulting image clustering performed by the IT2FPCM algorithm for 5 clusters to Figure 2(e) because of the gray levels containing the image.
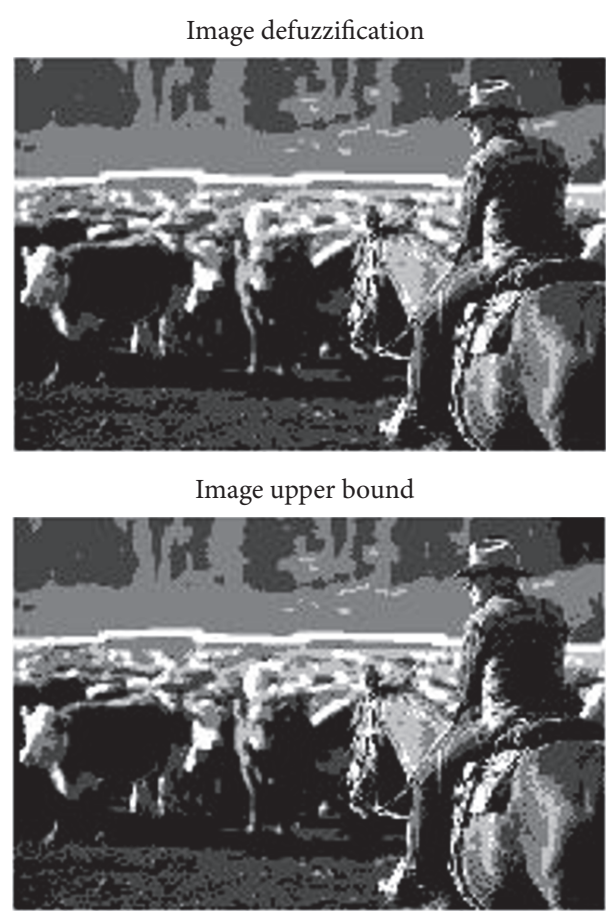
(15), to prove that the proposed method is better with a significant difference with respect to the other existing methods. Statistical tests were performed with the number of clusters that each validation index indicates as the best; in these statistical tests for the datasets and images we can observe that $69.45 \%$ of the hypothesis tests performed with the different indices of validation are affirming the alternative hypothesis based on (15), and $30.55 \%$ of the hypothesis tests reject the alternative hypothesis.

It is noteworthy that the parameters used in this work are not the optimal ones for both algorithms, and to find the optimal parameters for both algorithms used in this work we can use optimization algorithms like in [33]. We can use the PSO, GSA, and GA algorithms among others, in order to improve the performance and automate the interval type- 2 clustering algorithms that were used.

\section{Competing Interests}

The authors declare that there is no conflict of interests regarding the publication of this paper.

\section{References}

[1] W. E. Phillips, R. P. Velthuizen, S. Phuphanich, L. O. Hall, L. P. Clarke, and M. L. Silbiger, "Application of fuzzy cmeans segmentation technique for tissue differentiation in MR images of a hemorrhagic glioblastoma multiforme," Magnetic Resonance Imaging, vol. 13, no. 2, pp. 277-290, 1995.

[2] M.-S. Yang, Y.-J. Hu, K. C.-R. Lin, and C. C.-L. Lin, "Segmentation techniques for tissue differentiation in MRI of ophthalmology using fuzzy clustering algorithms," Magnetic Resonance Imaging, vol. 20, no. 2, pp. 173-179, 2002.

[3] K. Hirota and W. Pedrycz, "Fuzzy computing for data mining," Proceedings of the IEEE, vol. 87, no. 9, pp. 1575-1600, 1999.

[4] J. C. Bezdek, Pattern recognition with fuzzy objective function algorithms, Plenum Press, NY, USA, 1981.

[5] N. S. Iyer, A. Kandel, and M. Schneider, "Feature-based fuzzy classification for interpretation of mammograms," Fuzzy Sets and Systems, vol. 114, no. 2, pp. 271-280, 2000.

[6] X. Chang, W. Li, and J. Farrell, "C-means clustering based fuzzy modeling method," in Proceedings of the 9th IEEE International Conference on Fuzzy Systems (FUZZ-IEEE '00), vol. 2, pp. 937940, May 2000.

[7] R. Krishnapuram and J. M. Keller, "A possibilistic approach to clustering," IEEE Transactions on Fuzzy Systems, vol. 1, no. 2, pp. 98-110, 1993.

[8] N. R. Pal, K. Pal, and J. C. Bezdek, "A mixed c-means clustering model," in Proceedings of the 6th IEEE International Conference on Fuzzy Systems, vol. 1, p. 11, IEEE, Barcelona, Spain, July 1997.

[9] N. N. Karnik and J. M. Mendel, "Operations on type-2 fuzzy sets," Fuzzy Sets and Systems, vol. 122, no. 2, pp. 327-348, 2001.

[10] J. Mendel, Uncertain Rule-Based Fuzzy Logic Systems: Introduction and New Directions, Prentice-Hall, 2001.

[11] F. C.-H. Rhee and C. Hwang, "A type-2 fuzzy C-means clustering algorithm," in Proceedings of the Joint 9th IFSA World Congress and 20th NAFIPS International Conference, pp. 19261929, British Columbia, Canada, July 2001.

[12] M. H. Fazel Zarandi, M. Zarinbal, and I. B. Turksen, “TypeII fuzzy possibilistic C-Mean clustering," in Proceedings of the
Joint International Fuzzy Systems Association World Congress (IFSA '09) and European Society of Fuzzy Logic and Technology Conference (EUSFLAT '09), pp. 30-35, July 2009.

[13] M. H. F. Zarandi, S. M. Golsefid, and S. Bastani, "Dual centers fuzzy type-2 clustering," in Proceedings of the 9th Joint World Congress on Fuzzy Systems and NAFIPS Annual Meeting (IFSA/NAFIPS '13), pp. 1215-1220, Alberta, Canada, June 2013.

[14] E. Rubio and O. Castillo, "Designing type-2 fuzzy systems using the interval type-2 fuzzy C-means algorithm," in Proceedings of the International Seminar Computing Intelligence (ISCI '13), Tijuana, Mexico, November 2013.

[15] Y.-H. Byeon and K.-C. Kwak, "Knowledge discovery and modeling based on conditional fuzzy clustering with interval Type-2 fuzzy," in Proceedings of the 7th International Joint Conference on Knowledge Discovery, Knowledge Engineering and Knowledge Management (IC3K '15), pp. 440-444, Lisbon, Portugal, November 2015.

[16] L. Yu, J. Xiao, and G. Zheng, "Robust interval type-2 possibilistic c-means clustering and its application for fuzzy modeling," in Proceedings of the 6th International Conference on Fuzzy Systems and Knowledge Discovery (FSKD '09), pp. 360-365, IEEE, Tianjin, China, August 2009.

[17] O. Obajemu, M. Mahfouf, and L. A. Torres-Salomao, "A new interval type-2 fuzzy clustering algorithm for interval type-2 fuzzy modelling with application to heat treatment of steel," IFAC Proceedings, vol. 47, no. 3, pp. 10658-10663, 2014.

[18] B.-I. Choi and F. C.-H. Rhee, "Interval type-2 fuzzy membership function generation methods for pattern recognition," Information Sciences, vol. 179, no. 13, pp. 2102-2122, 2009.

[19] E. Rubio and O. Castillo, "Interval type-2 fuzzy clustering for membership function generation," in Proceedings of the 2013 IEEE Workshop on Hybrid Intelligent Models and Applications (HIMA '13), pp. 13-18, IEEE, Singapore, April 2013.

[20] L. Tlig, M. Sayadi, and F. Fnaeich, "A new descriptor for textured image segmentation based on fuzzy type- 2 clustering approach," in Proceedings of the 2nd International Conference on Image Processing Theory, Tools and Applications (IPTA '10), pp. 258263, Paris, France, July 2010.

[21] M. H. F. Zarandi and M. Zarinbal, "A new image enhancement method type-2 possibilistic C-mean approach," in Proceedings of the 2013 Joint IFSA World Congress and NAFIPS Annual Meeting (IFSA/NAFIPS '13), pp. 1131-1135, June 2013.

[22] R. Ceylan, Y. Özbay, and B. Karlik, "A novel approach for classification of ECG arrhythmias: type-2 fuzzy clustering neural network," Expert Systems with Applications, vol. 36, no. 3, part 2, pp. 6721-6726, 2009.

[23] M. A. Raza and F. C.-H. Rhee, "Interval type-2 approach to kernel possibilistic C-means clustering," in Proceedings of the IEEE International Conference on Fuzzy Systems (FUZZ-IEEE '12), pp. 1-7, Brisbane, Australia, June 2012.

[24] D. D. Nguyen and L. T. Ngo, "Multiple kernel interval type-2 fuzzy c-means clustering," in Proceedings of the IEEE International Conference on Fuzzy Systems (FUZZ-IEEE '13), pp. 1-8, Hyderabad, India, July 2013.

[25] M. U. Nguyen, L. T. Ngo, and T. T. Dao, "Improved interval type-2 fuzzy subtractive clustering for obstacle detection of robot vision from stream of depth camera," in Proceedings of the 12th International Conference on Intelligent Systems Design and Applications (ISDA '12), pp. 903-908, November 2012.

[26] S. M. Golsefid, M. H. F. Zarandi, and S. Bastani, "Fuzzy type-2 c-ellipses clustering," in Proceedings of the IFSA World Congress 
and NAFIPS Annual Meeting (IFSA/NAFIPS '13), pp. 1221-1226, June 2013.

[27] S. M. M. Golsefid and M. H. Fazel Zarandi, "Dual-centers type2 fuzzy clustering framework and its verification and validation indices," Applied Soft Computing Journal, vol. 47, pp. 600-613, 2016.

[28] E. Rubio, O. Castillo, and P. Melin, "A new validation index for fuzzy clustering and its comparisons with other methods," in Proceedings of the IEEE International Conference on Systems, Man, and Cybernetics (SMC '11), pp. 301-306, October 2011.

[29] J. Yen and R. Langari, "Fuzzy logic in pattern recognition," in Fuzzy Logic: Intelligence, Control, and Information, pp. 351-377, Prentice Hall, Upper Saddle River, NJ, USA, 1999.

[30] M. K. Pakhira, S. Bandyopadhyay, and U. Maulik, "A study of some fuzzy cluster validity indices, genetic clustering and application to pixel classification," Fuzzy Sets and Systems, vol. 155, no. 2, pp. 191-214, 2005.

[31] B. Rezaee, "A cluster validity index for fuzzy clustering," Fuzzy Sets and Systems, vol. 161, no. 23, pp. 3014-3025, 2010.

[32] K.-L. Wu and M.-S. Yang, "A cluster validity index for fuzzy clustering," Pattern Recognition Letters, vol. 26, no. 9, pp. 12751291, 2005.

[33] E. Rubio and O. Castillo, "Optimization of the interval type-2 fuzzy C-means using particle swarm optimization," in Proceedings of the World Congress on Nature and Biologically Inspired Computing (NaBIC '13), pp. 10-15, IEEE, August 2013. 

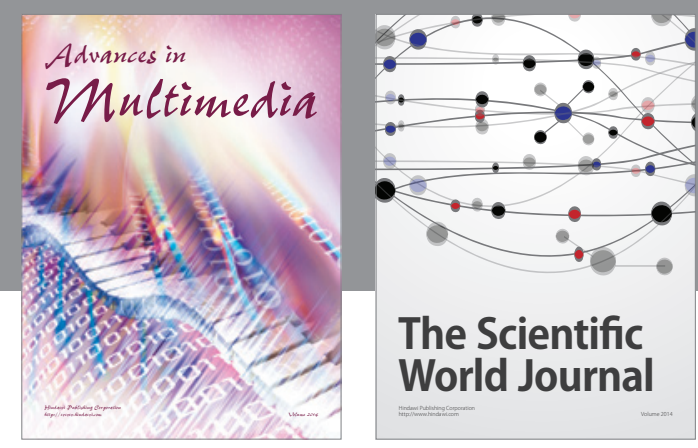

The Scientific World Journal
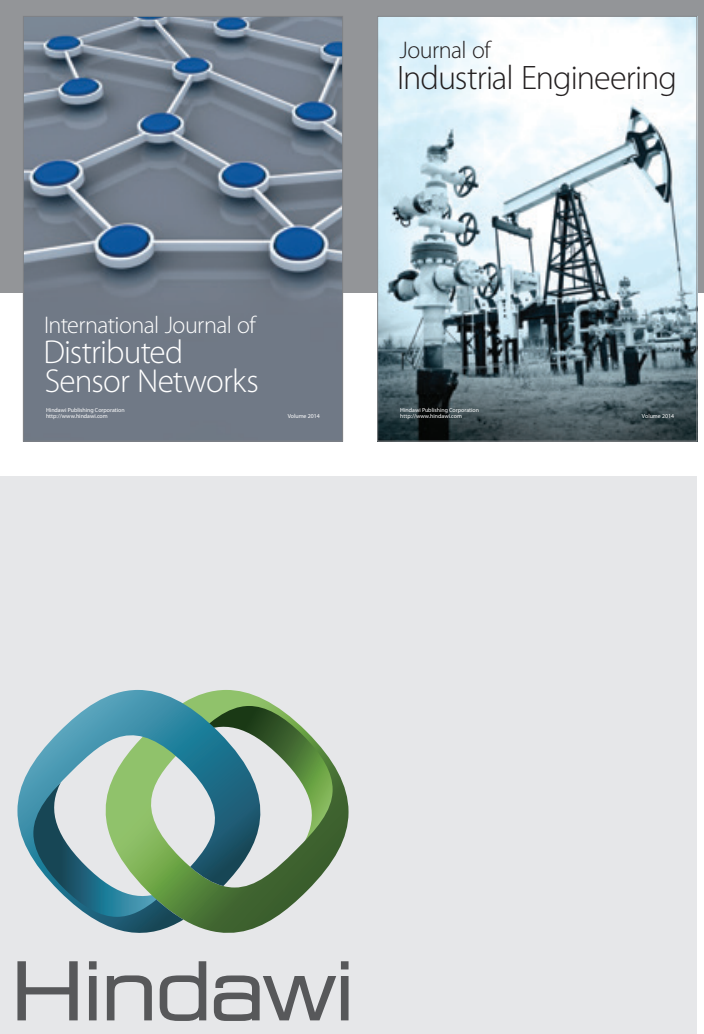

Submit your manuscripts at

https://www.hindawi.com

\section{Computer Networks} and Communications
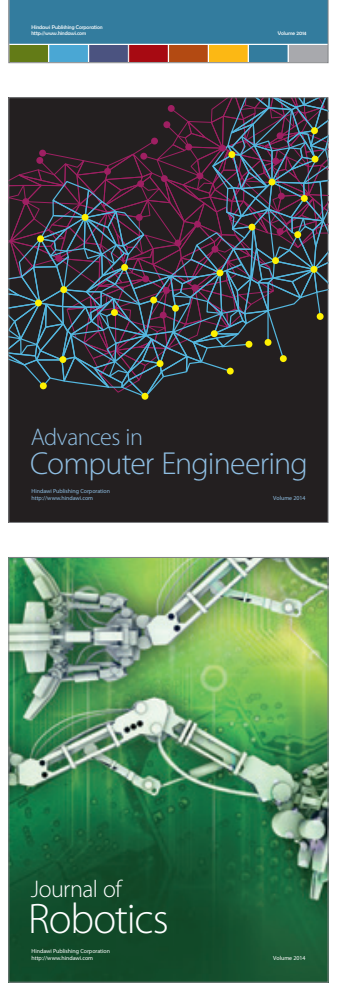
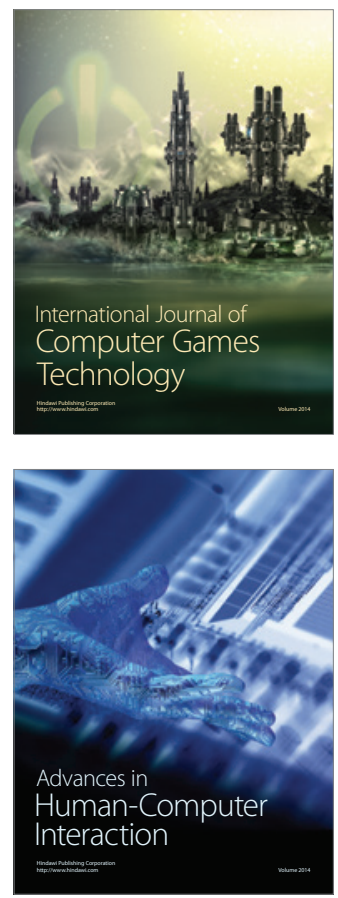
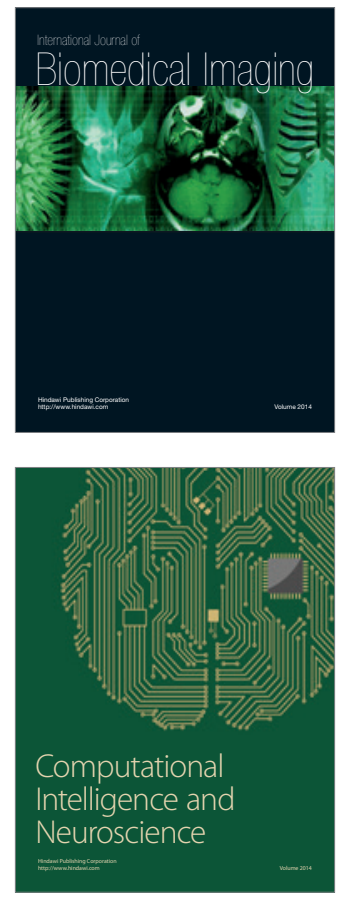
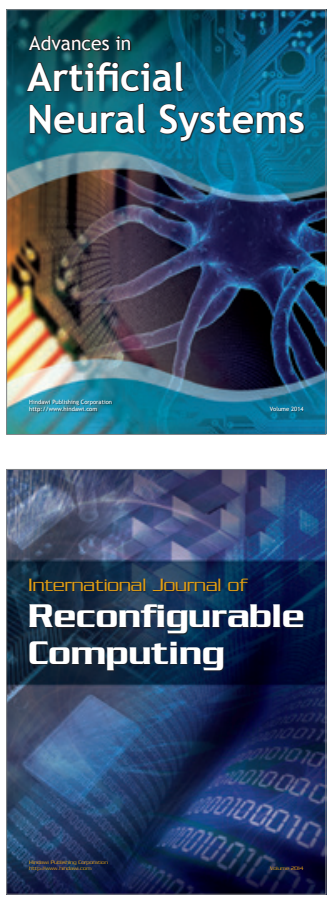
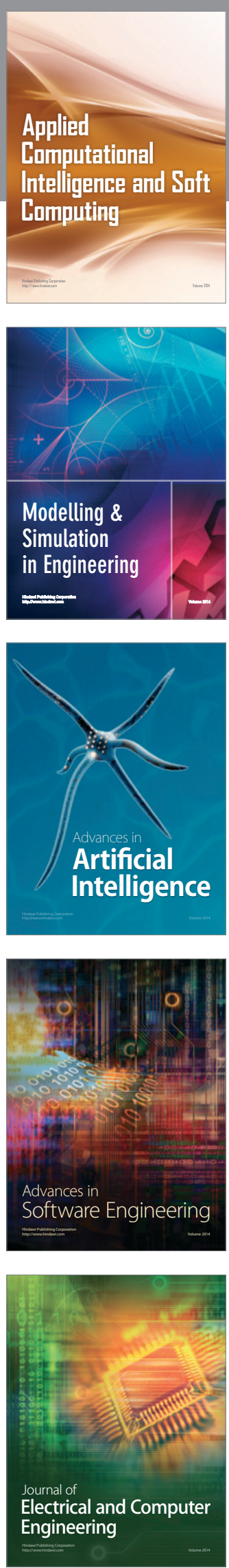